\title{
Urban Evolution as a Spatio-Functional Interaction Process: The Case of Central Shanghai
}

\author{
Yao Shen, Kayvan Karimi \\ y.shen.12@ucl.ac.uk_k.karimi@ucl.ac.uk \\ Space Syntax Laboratory, Bartlett School of Architecture, University College London \\ 22 Gordon St, Bloomsbury, \\ London WC1H 0QB, UK
}

1 May 2017

\begin{abstract}
Precisely identifying the shifting urban spatial structures produced by urban forms and functions contributes to an advanced understanding of urban morphological dynamics and related planning practices. Although evolutionary city structures have been widely discussed, comprehensive research focusing on the dynamic interactions between spatial and functional sub-systems is still lacking. This article investigates the transformation of urban centrality structures as captured by the shifting interdependence between centrality indices (angular integration and choice) and delivered urban function connectivity metrics (accessible function density, diversity and cognitive distance), generated in tandem by spatial network and land-use patterns. By reconceptualising urban evolution as a centrality process in which spatial and functional centrality processes co-exist and co-evolve, this study constructs a systematic connection between these two types of centrality structures during identical transition periods in Shanghai's history of modernisation. Four critical snapshots of street networks and Points-of-Interest (POIs) in history are selected as a spatiotemporal description of the urban transformation of Central Shanghai. The results demonstrate that the centrality structures hidden behind the spatial network and land-use distributions have historically affected each other dynamically. Certain degrees of inconsistency are observed between these two systems, and the characteristics of urban developments at various stages can be distinguished according to
\end{abstract}


modes of spatio-functional interaction at multi-scales. The findings of the canonical discriminant analysis indicate that shifting complex interrelationships between the spatial network and land-use patterns are the major determinants of the formation of the urban function regions. The proposed framework offers valuable insights into the morphological evolution process of cities as indicated by the configurational interplay between form and function, and it represents a novel way to explicitly identify urban change.

Keyword: urban evolution, spatio-functional interaction, land-use distribution, centrality, space syntax,

\section{INTRODUCTION}

Depicting urban spatial structures is one vital prerequisite to uncovering the process by which urban morphologies evolve. An urban spatial structure is a sum of the various ways in which people interact through perceivable urban forms (Anas et al. 1998). A centrality structure reflecting the sense of 'a centre' usually refers to the concentration of urban activities in a prominent location (Horton and Reynolds 1971; Hillier 1999). In this sense, an urban structure generally contains the functional elements representing the interactions between form and function that, in turn, illustrate the spatial conditions facilitating the morphological agglomeration of land uses. Revealing the links between the spatial and functional elements of the urbanisation process is vital for any urban revitalisation programme, as it contributes to sustaining the long-term prosperity of city centres (Burger and Meijers 2012; Tallon 2013).

In traditional typo-morphological research, the built environment is analogised to an architectural biosphere, and urban spatial elements - buildings, blocks, streets, etc. are considered 'species' with distinguishable characteristics. Thus, tracing the transformation of the spatial typologies of urban form has historically been the main approach to understanding hidden spatio-social transformations (e.g., Moudon 1997; Pinchemel and Pinchemel 1983; Darin 1998; Cataldi et al. 2002; Pinho and Oliveira 2009). With their emphasis on the structural properties of spatial configurations, configurational studies focus on the formulation and reproduction of hierarchical urban 
centres as driven by the spatial interactions of spatial elements at various scales (e.g., Hillier 1999). Numerous studies have demonstrated that changes in spatial centrality generated by the urban grid dramatically influence the distributed commercial amenities (e.g., Hillier 1999; Porta et al., 2009; Porta et al., 2012; Scoppa \& Peponis, 2015) The land-use patterns identified in configurational studies and space syntax studies, in particular, are considered to be the products of spatial centrality, which drives the economic process (Hillier 1996). This argument reclaims the theoretical and practical positions of urban design in the land-use allocation process. However, the inherent structures of the land-use patterns are over-simplified, thereby constraining the further exploration of how land-use locations react to the spatial advantages provided by the spatial network.

A land-use system is not simply a layer that corresponds to the spatial network. Rather, it has its own logic by which it formulates observable patterns. It can be impacted by the bid price from the central area to the periphery (Alonso 1960), competition or market sharing (Hotelling 1990; Christaller 1996), complementary interaction (e.g., Eppli and Shilling 1996), gravity interaction (e.g., Wilson 1998), investment in the transport system (Wegener 2004; Waddell 2002), and the geometrical accessibility to other landuses (Stahle et al. 2005; Sevtsuk 2010; Agryzkov et al. 2016). Conventional methods of identifying the shapes of urban centres are based on the analysis of land-use agglomeration with reviews of informed opinion (e.g., Batty et al., 1997; Wheaton 1974). In contrast to how space syntax theory explains the process of urban evolution, geographical studies generally consider urban evolution to be a process of accessibility change at larger scales, driven by a series of land-use changes resulting from underlying socioeconomic transformation; however, these studies neglect the role of urban geometrical structure (Geurs and Wee 2004; Batty 2009). Recent efforts have aimed to describe land-use interaction through high-resolution morphological analyses of the built environment. For example, using spatially lagged logistic regression methods, Sevtsuk (2014) has found that the locational decisions of particular types of retailers in buildings along street networks are related to the accessibility of other types of commercial amenities. On the basis of the social media data gathered from Foursquare and Twitter, Agryzkov et al. (2016) visualised city as a complex network, and adopted the PageRank algorithm to measure its node centrality with the aim of assessing the successfulness of public space. 
To summarize, spatial and functional centrality structures are both vital dimensions of the definition of an urban centre and its hierarchical nature. Nevertheless, existing studies have failed to apply a novel, systematic approach to measuring and comparing them from a syntactic/structural perspective on the urban evolutionary process. To address this gap, this article applies a spatio-functional model to investigate the extent to which the structure of urban centrality is explained by the distribution of land-use through the spatial network and the process by which the spatial network interacts with urban functions over the stages of urbanisation. There are three aims of this research. The first is to examine the extent to which the functional centrality structures indexed by urban function connectivity metrics differ from the spatial centrality structures as defined by space syntax centrality measures in the history of urbanisation. The second is to explore the extent to which these two types of centrality structures correlate with each other over time. The final aim is to investigate the extent to which this spatiofunctional relationship performs differently from one location to another.

\section{METHOD AND DATA}

\subsection{Research design}

The research framework used in this article is illustrated in Figure 1. Four main modules are used to conduct this investigation. The first module is data processing (a) to convert all the obtained historical information into geocoded datasets. The street networks shown on historical maps are digitised as the spatial layers of segmental maps, and locational information on land-uses and their place significance in local atlases, local planning chronicles, and other data resources are gathered and geocoded along the street segments where they are located. In the following two steps ( $b$ and $c$ ), which compute both spatial and functional centrality measures during different periods, urban evolution is conceptually defined as both a spatial centrality process and a functional centrality process, respectively. The last module (d: spatio-functional centrality process) explores the descriptive statistics, inter-scale/inter-centrality correlations and raster-based centrality change with the aim of capturing the differentiated performance of these two types of urban centrality processes quantitatively and qualitatively. Finally, a canonical discriminant analysis is conducted to quantitatively identify the predictive accuracy of 
the space syntax centrality and the urban function connectivity variables in various scenarios to delineate the detected urban function regions.

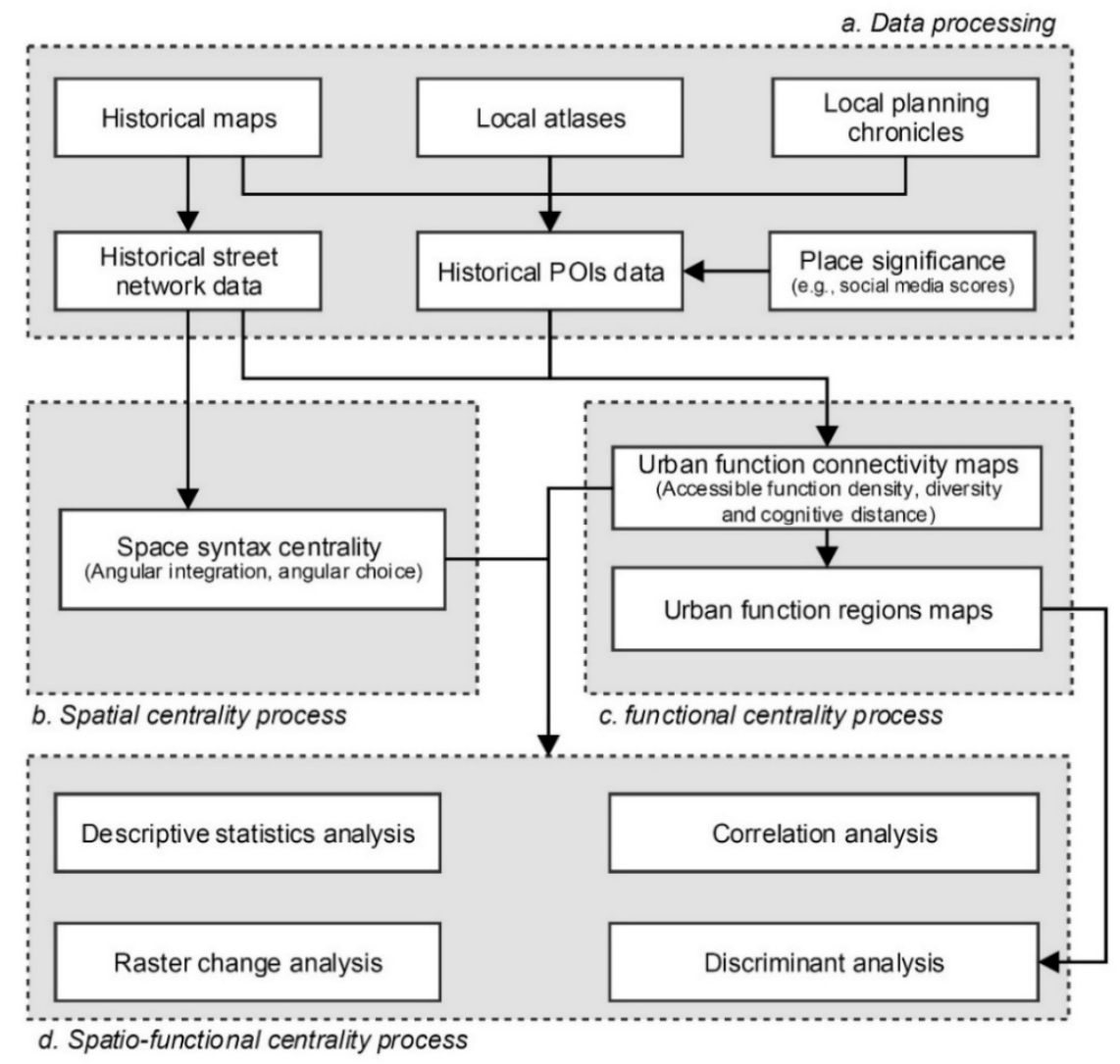

Figure 1. The proposed framework for analysing urban transformation.

\subsection{The method}

\subsubsection{Centrality computation}

Street centrality measures based on a graph representation of the built environment can provide finely-scaled maps of urban spatial structures with non-Euclidean settings. Two groups of network centrality measures are used in this study: the spatial accessibility measures that serve as the space syntax centrality indices, including integration and choice, and the urban function connectivity indices, including density, diversity and cognitive distance. These three key aspects of urban function layouts originate from the processes of densification, diversification (Cervero and Kockelman 1997), and cost- 
saving (or efficiency maximisation (Penn and Turner 2004)), driven by the location decisions of economic agglomeration (Fujita et al. 1999), which would shift the centrality structures of urban activities in the city. The functional densification and diversification represent the size and variety of attraction concentration based on metric distance metrics, whilst the cognitive distance describes the overall geometrical cost for the land-use concentration. A successful combination of these three elements captures the geometrical concentration of active land-uses comprehensively.

Typical radii, including $500 \mathrm{~m}, 1,000 \mathrm{~m}, 2,500 \mathrm{~m}, 5,000 \mathrm{~m}$, and 10,000 m, are used to represent spatial and functional accessibilities across scales. In our experiments, these radii can successfully summarise urban structural centrality at various scales by controlling both information redundancy and boundary effects.

\section{1) Street-based spatial centrality - space syntax centrality indices}

The so-called street-based spatial centrality indices are computed according to space syntax theory and include both segmental angular integration and choice (Turner 2001; Hillier and Iida, 2005; Hillier et al., 2012). Angular integration can be understood as an adjusted closeness in graph analysis and represents the angular shallowness between segments, which can be formally defined as follows:

$\operatorname{INT}_{(i, r)}=\frac{\left(N_{i}-1\right)}{\sum_{j=1}^{J} \operatorname{Dep}_{(i, j)}},\left\{\operatorname{dis}_{(i, j)} \leq r\right\}$

In this equation, the angular integration $\left(I N T_{(i, r)}\right)$ at the radius $\mathrm{r}$ is represented as the mean angular depth $\left(\operatorname{Dep}_{(i, j)}\right)$ from segment $i$ to all the reachable segments $j$ within a buffer zone defined by the pre-identified radius $r$.

The so-called angular choice index $(\mathrm{CHO})$ in the space syntax model is very similar to the concept of betweenness in the graph analysis but uses the angular change at each road intersection as the expenditure to find the shortest path (Turner 2001). It can be defined as the number of times $\left(n_{j k}\right)$ that the focused segment $i$ has been passed through in the angular shortest paths from segment $\mathrm{j}$ to segment $\mathrm{k}$ within a reachable area defined by a fixed radius $r$. From a mathematical perspective, this definition can be represented as follows:

$\mathrm{CHO}_{(i, r)}=\sum_{k=1}^{K} n_{j k},\left\{d i s_{(i, j)} \leq r ; d i s_{(i, k)} \leq r\right\}$ 


\section{2) Street-based functional centrality - urban function connectivity metrics}

The accessible function density index (DEN) measures the accumulation of scored urban activities from each street within a defined radius through the shortest reachable paths. Assuming there are $\mathrm{K}$ types of active land-uses, the accessible function density for the segment node i at radius $\mathrm{r}$ would be aggregated as $D E N_{(i, r)}$ :

$\operatorname{DEN}_{(i, r)}=\sum_{k=1}^{K} \sum_{j=1}^{J} O_{(j, k)} \times W_{(j, k)}, \quad\left\{d i s_{(i, j)} \leq r\right\}$

This summation takes into account the function nodes assigned to street segment edges and weighted based on social media check-in scores. In the equation above, $r$ is the defined radius, $O_{(j, k)}$ refers to the potential opportunities that can be supplied, and $W_{(j, k)}$ is the specified weight of the function node $\mathrm{j}$ in type $\mathrm{k}$, used to reflect its popularity. In this study, we use the normalised number of social media check-ins assigned to each POI as its weight on the demand side, which can be presented as $W_{(j, k)}=\frac{\log C_{(j, k)}}{\log C_{k}^{\max }}$, where $\log C_{(j, k)}$ represents the log-normalised check-ins for the specific function node $\mathrm{j}$ in type $\mathrm{k}$, and $\log C_{k}^{\max }$ denotes the $\log$-normalised value of the maximum check-ins for all the function nodes. Because we lack, for the purpose of calibration, reliable corresponding information about land-use from the supply perspective, it is assumed that the supply of the function is even across locations.

It has been argued that social media check-in data only capture the subsets of urban population, thereby causing potential bias of samples and producing misleading conclusions (e.g., Duggan and Brenner 2013). A consensus has been established is that the applicability of social media dataset should be carefully verified (Ruths and Pfeffer 2014). In this article, we propose to use normalised social media check-in scores to present the popularity of an urban amenity. This idea has been validated through the hedonic modelling of house price variation with the weights of social media check-ins in favourable functions in people's daily life (Shen and Karimi 2016). These findings inform that in contemporary society, the amenities with small service areas may be very popular on the social media landscape, and vice versa. In this sense, social media check-in scores are a type of unique data indicating the more 'real' sense of function in human's mind rather than conventionally used weighting parameters, such as floor area.

The accessible function diversity index (DIV) measures the balance degree of all weighted urban activities reachable from the original street within a given radius. Information entropy is applied to measure the diversity of urban function nodes from 
segment node $\mathrm{i}$ at radius $\mathrm{r}$ and is represented as $D I V_{i, r}$. A normalisation transformation is applied to make the different types of activities comparable by converting the absolute density to a relative density; this is accomplished by dividing the accessible weighted density in type $\mathrm{k}$ for each segment node by the maximum value of the accessible density of land-use of the same type at the same radius for all the segment nodes within the study area $\left(\operatorname{NaDEN} N_{(i, k, r)}=\frac{D E N_{(i, k, r)}}{\left.D E N_{(k, r)}^{\max }\right)}\right.$. The presence probability $\left(P_{(i, k, r)}\right)$ of the function nodes in type $\mathrm{k}$ at radius $\mathrm{r}$ for segment node $\mathrm{i}$ is measured by its empirically observed frequency of normalised density $\left(\operatorname{NaDEN} N_{(i, k, r)}\right)$ among all $\mathrm{K}$ types of land-uses.

The computation of accessible diversity can be formally represented as follows.

$$
\begin{aligned}
& \operatorname{DIV}_{(i, r)}=\frac{-\sum_{k=1}^{K} P_{(i, k, r)} \times \ln \left(P_{(i, k, r)}\right)}{\ln (K)}, \quad\left\{d i s_{(i, j)} \leq r\right\} \\
& P_{j k, r}=\frac{\operatorname{NaDEN}_{(i, k, r)}}{\sum_{k=1}^{K} \operatorname{NaDEN}_{(i, k, r)}} \ldots \ldots \ldots \ldots \ldots \ldots \ldots \ldots \ldots \ldots \ldots \ldots \ldots \ldots \ldots
\end{aligned}
$$

The cognitive distance index (DIS) measures the mean angular step depth to all the reachable urban activities from the original street within a given radius through the shortest paths. It reveals the cognitive efforts required to reach all accessible functions from the original street segments beyond the same energy expenditure that is measured in light of the metric length of the streets. This index can be formally expressed in the following equation in which, $D e p_{(i, j, k)}$ shows the angular step depth from segment node $\mathrm{i}$ to function node $\mathrm{j}$ in type $\mathrm{k}$ within the buffer area defined by radius $\mathrm{r}$, and $N_{(i, r)}$ is the summation of the accessible functions at the same radius.

$D I S_{(i, r)}=\frac{\sum_{k=1}^{K} \sum_{j=1}^{J} D e p_{(i, j, k)}}{N_{(i, r)}},\left\{d i s_{(i, j)}<r\right\}$

The integrated urban function connectivity index (UFC) is an integrated measurement that measures the degree to which the dense and diverse urban activities are accessible with less angular step depth within a given radius. Here, three principal dimensions reflecting the impacts of opportunity accumulations, function composition and cognitive distance, are incorporated into the final UFC index $\left(U F C_{(i, r)}\right)$, which can be calculated formally as follows:

$$
U F C_{(i, r)}=\frac{D E N_{(i, r)}{ }^{D I V}(i, r)}{D I S_{(i, r)}},\{\operatorname{dist}(i . j) \leq r\}
$$




\section{3) Street-based urban functional region structure}

Urban streets are connected to different types of functions, in which the UFRs are characterised by the function connectivity of different land-use types. In this sense, we apply the statistical data mining approach to quantitatively measure the similarity of the composition of function connectivity. Specifically, we use a k-means clustering analysis to partition the urban street segments and then annotate each cluster according to the unique composition of function connectivity.

The function angular closeness index (FAC) is a particular form of function connectivity that focuses on the specified type of land-use, and it measures the angular agglomeration of an urban function of a certain type through the shortest reachable urban paths within a given radius. The computational logic of this index is based on the idea of establishing so-called angular closeness, which is computed as the quotient in which node counts are divided by the mean angular step depth in the space syntax model. Mathematically, this metric can be identified in a straightforward way as follows.

$F A C_{(i, k, r)}=\frac{D E N_{(i, k, r)}}{D I S_{(i, k, r)}},\{\operatorname{dist}(i, j) \leq r\}$

In the equation above, $D E N_{(i, k, r)}$ refers to the accessible density of function nodes in type $\mathrm{k}$ from segment node $\mathrm{i}$ at the radius $\mathrm{r}$, and $D E F_{(i, k, r)}$ captures the angular delivery efficiency of these functions.

In this study, k-means clustering for several states is employed by using the FACs of each street as the vectors' dimensions due to its efficiency in handling large-sized numerical datasets (Bishop 2006). As its name implies, k-means clustering intends to group objects into predefined k clusters where every object in the same cluster will have the nearest mean. Consequently, the objective of k-means clustering in this study is to minimise the total intra-cluster variance, which is measured by the squared errors. As a type of iterative descent clustering algorithm, k-means clustering can be summarised as follows:

$J_{(c, r)}=\min _{C} \sum_{l=1}^{L} \sum_{C(i)=l} \operatorname{dist}\left(V_{i}, \bar{V}_{l}\right),\{\operatorname{dist}(i, j) \leq r\}$

where $J_{(c, r)}$ is an objective function for a given cluster assignment $C$ at a radius $\mathrm{r}$, $C(i)$ refers to the label of the observations, $\bar{V}_{l}$ is the mean vector for the 1 th cluster, and $V_{i}$ is a multi-dimensional vector illustrating the co-presence of function accessibility of 
various land-uses $\left(V_{i} \in\left(F A C_{(i, 1, r)}, F A C_{(i, 2, r)}, \ldots \ldots, F A C_{(i, K, r)}\right)\right)$. This process will be repeated iteratively until the grouping results are stable with a minimised sum of squares. In the current study, Dunn's Index and the Silhouette Index are applied as validation measurements to evaluate the most appropriate number of clusters. The radius for identifying the functional region is the walking distance of $1,000 \mathrm{~m}$.

\section{4) Classification of land-use patterns}

Active land-uses in this study are defined as the complementary land-uses that are more likely to be linked by urban travels and thereby contribute to emergent movement patterns. Unlike mixed-use developments, which seek a balance of all land-uses, the active uses in this study are based on function complementarity between non-residential land-uses (Hess et al. 2001). Complementary land-uses (active land-uses in this article) include retail, catering, hotels, offices, schools, social services, hospitals, recreation, culture, parks and transport, according to the main activity types that are distinguished in social media.

\subsubsection{2 other analyses}

Because of some offset and deflection between the historical street network datasets, the most effective way to calculate the change in one fixed type of centrality at the same scale is to use the raster calculation with a spatial tolerance. A $150 \mathrm{~m}$ buffer zone is generated for each street segment, and then the centrality values assigned to streets are used as the sample points to interpolate the centrality surface. The change rate of the centrality surface is calculated as the discrepancy between the normalised centrality surfaces at different time nodes.

To validate the influence of the spatio-functional centrality measures on predicting the membership of the detected functional regions, a canonical discriminant analysis (CDA) is employed. It first reduces the dimensions of the input variables by packaging them in $\mathrm{N}-1$ functions, and $\mathrm{N}$ here denotes the total number of memberships in the sample. CDA evaluates the predictive accuracy based on these discriminating functions by comparing the predicted results and the sample. As a method of supervised learning, CDA provides opportunities to assess the explanatory power of a set of factors with respect to the grouping phenomenon. 


\subsection{Data}

\subsubsection{Study area}

This analysis focuses on Central Shanghai in the Shanghai Metropolitan Area (SMA) as the setting for our empirical study. Shanghai has been China's economic centre since 1949 and is currently one of the country's largest municipalities, along with Beijing, Tianjin, and Chongqing. Because of its geographical location, Shanghai was the first city in China to undergo modernisation after the Second Opium War when Shanghai was a semi-colonial city. Since the Open Door Policy was implemented in 1979, Shanghai has been significantly growing and has been transformed into a mega-city. The central area in SMA serves as an important case study for this article because of the complexity of its built environment and because it represents other Chinese cities undergoing rapid urban growth.

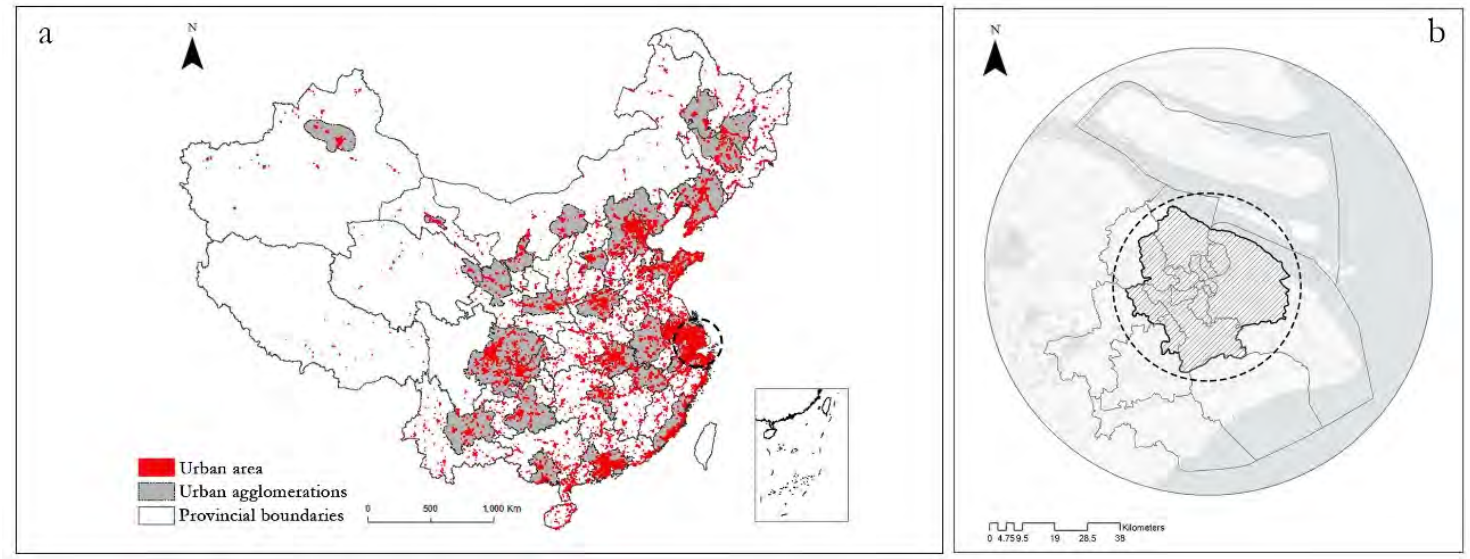

Figure 2. Study areas in the Shanghai Metropolitan Area (SMA): (a) the location of Shanghai City in the Chinese city system; (b) the location of the study area in the SMA

\subsubsection{Street network - spatial network}

The street network datasets applied in this article are redrawn according to the historical maps. To produce accurate segmental maps, the street network data are generated by converting the axial maps to the segmental maps. Unlike those produced directly by the central road lines, the segmental maps in this study are produced by controlling the axiality of segments, which reflects the minimal cognitive cost to people travelling from one segment to another. The final datasets are shown in Figure 3. 


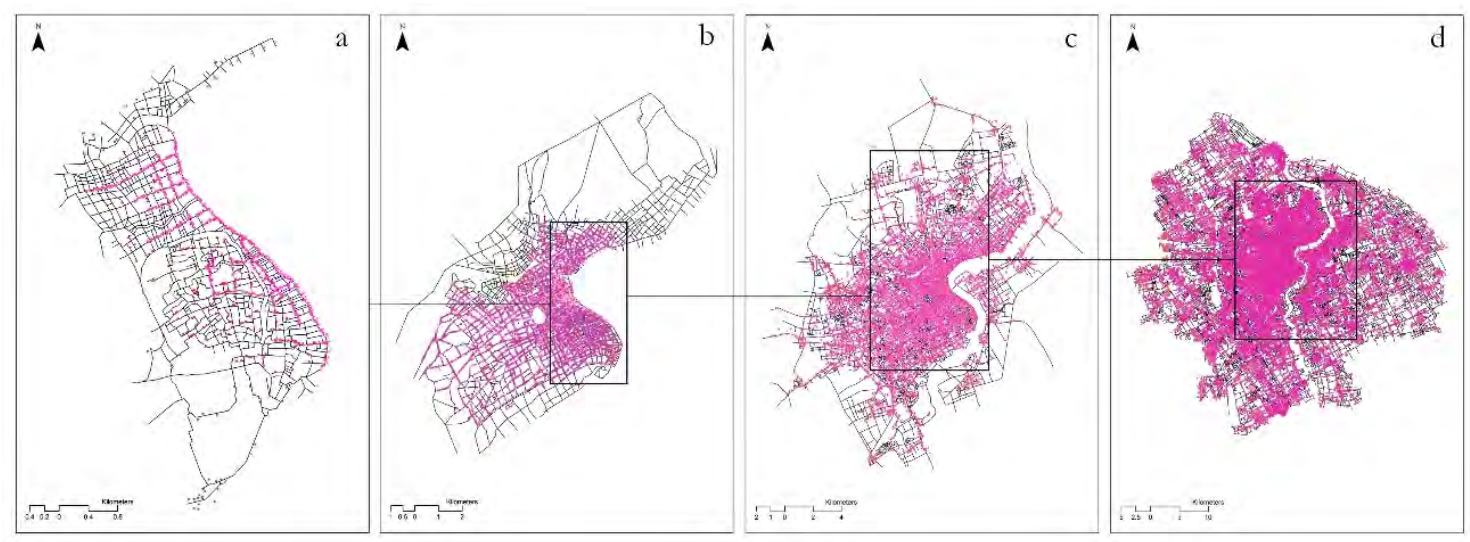

Figure 3. Geocoded historical POIs and associated street networks during the 1880 s (a), 1940s (b), 1980s (c), and 2010s (d).

\subsubsection{Historical POIs - land-use patterns}

Land-use data can be represented and summarised in many ways with various settings, which can lead to different observations. POI datasets are the fine-grained land-use patterns with identical relations to the spatial network. In this study, the land-use locations on the historical maps, and in local atlases and local planning chronicles (Sun et al. 1999; Cheng and $\mathrm{Wu}$ 2004; Shanghai Institute of Surveying and Mapping 1989), have been digitised, labelled, geocoded, and assigned to the street segments where they were historically located.

Although the significance of various types of land-uses might vary historically, a generalised but rigorous classification of urban functions is a prerequisite for conducting an objective comparison of different snapshots of historical urbanisation. In this article, check-in behaviours (including check-in rate - the proportion of checked in POIs in all POIs, the number of check-ins per POI and the amount of checked-in social media users per POI) happened in various types of land-uses were analysed to produce a volunteered classification of urban amenities. Thus, 256 sub-types of land-uses have been categorised into 11 main complementary types by reducing information redundancy. This definition has been tested in other Chinese cities and its applicability was verified via statistical models on urban performance (Shen and Karimi 2016). To maintain a consistent classification of land-use distributions during different periods, all historical POIs have been classified into these 11 types of active land-use that are complementary to each other (Table 1). 
The sizes of the POI datasets have become increasingly larger with urban development. However, the POI dataset from the 1940s is larger than the dataset of POIs in the 1980s due to the map resolution used by its cartographers. There are also some data missing from the current POI datasets proposed for use in this study. The detailed data on retail and catering activities in Central Shanghai in the 1880s are not available in this dataset. Therefore, they have been merged into a new category of land-use named commercial establishment (COM). Their densities are estimated using the lengths of the frontages of the high streets reported in the local planning chronicles. Social media checkin features in the POI data are used as the weights for each amenity in the 2010s, whereas the variation in the locational popularity of individual land-use locations is assumed to be equal during other periods because of the absence of relevant data in the historic land-use maps; the agglomeration of land-uses around a certain location is used to estimate that location's significance.

Table 1: Historical POI classification and numbers in Central Shanghai

\begin{tabular}{|c|c|c|c|c|c|c|c|c|}
\hline \multirow[t]{2}{*}{ Type } & \multirow[t]{2}{*}{ Abbreviation } & \multicolumn{4}{|c|}{ POIs count } & \multirow{2}{*}{$\begin{array}{c}\text { Check-in } \\
\text { Rate }\end{array}$} & \multirow{2}{*}{$\begin{array}{l}\text { Check-ins } \\
\text { per POI }\end{array}$} & \multirow{2}{*}{$\begin{array}{c}\text { Check-in } \\
\text { users per } \\
\text { POI }\end{array}$} \\
\hline & & $1880 \mathrm{~s}$ & $1940 \mathrm{~s}$ & $1980 \mathrm{~s}$ & $2010 \mathrm{~s}$ & & & \\
\hline Retail & RET & 8419 & 38,002 & 12,180 & 81,312 & $18.355 \%$ & 16.936 & 11.531 \\
\hline Catering & CAT & & 1,284 & 1,276 & 72,351 & $48.407 \%$ & 43.632 & 36.593 \\
\hline Hotel & HOT & 4 & 333 & 1,302 & 27,313 & $16.918 \%$ & 24.473 & 17.297 \\
\hline Office & OFF & 61 & 4,621 & 6,096 & 54,388 & $31.447 \%$ & 22.917 & 11.361 \\
\hline Education & EDU & 8 & 489 & 2,777 & 9,139 & $67.261 \%$ & 112.755 & 44.654 \\
\hline $\begin{array}{l}\text { Public } \\
\text { service }\end{array}$ & PUB & 43 & 1,855 & 1,949 & 10,762 & $80.002 \%$ & 16.239 & 10.237 \\
\hline Hospital & HOS & 12 & 748 & 438 & 3,518 & $90.421 \%$ & 149.723 & 82.204 \\
\hline Recreation & REC & 5 & 147 & 220 & 35,080 & $37.434 \%$ & 36.751 & 26.288 \\
\hline Culture & CUL & 123 & 236 & 94 & 20,068 & $4.739 \%$ & 24.695 & 17.671 \\
\hline Park & PAR & 7 & 75 & 77 & 4,250 & $68.894 \%$ & 83.691 & 57.549 \\
\hline \multirow[t]{3}{*}{ Transport } & TRA & 63 & 989 & 153 & 6,611 & $82.665 \%$ & 190.469 & 127.654 \\
\hline & & 1,167 & 48,779 & 27,562 & 324,792 & & & \\
\hline & $\begin{array}{r}\text { Weighted by } \\
\text { check-ins }\end{array}$ & $\mathrm{N}$ & $\mathrm{N}$ & $\mathrm{N}$ & $\mathrm{Y}$ & & & \\
\hline
\end{tabular}

I: Due to the absence of detailed information regarding retail and catering amenities, both types of amenities are merged into one group called commercial establishments (COM) and estimated according to the length of the high streets reported in the historical literature.

\subsection{Identification of urban transformation in Central Shanghai}

Shanghai City is used for the empirical studies in this article not only because it is typical of Chinese cities but also because it was one of the first modern cities in China to undergo 
significant spatial transformation. To clearly define the meanings of the selected snapshots of Shanghai's history of urbanisation, spatial features are used to objectively identify the critical steps in the city's urbanisation. Four metrics of urban form - average block size, road network density, total length, and total urban area - are used to capture various aspects of urban growth in relation to population growth. It is clear that, in parallel with rapid urban growth, Shanghai's urban form became less pedestrian-friendly, with larger blocks and lower road network density (Table 2). This trend, however, is not as consistent as expected. Between the 1940s and the 1980s, the speed of population growth in Shanghai slowed, as did the growth of the city's urban area, total street length, and block sizes (Figure 4). Its street network density, however, grew slightly during the same time interval, which suggests that the densification process of the road network was highly significant. This challenges the traditional definition of the stages of urbanisation, according to which the modern history of Chinese cities is divided into the early modernisation and modernisation periods using the birth of the People's Republic of China in 1949 as the turning point. Based on Shanghai's historical spatial configuration, this study redefines the key stages of the city's urbanisation process as follows: 'early rapid urban growth' is the period from the 1880 s to the 1940 s when the city grew rapidly due to the connection of the colonial areas to the historic centres; 'steady urban growth' is the period from the 1940 s to the 1980 s when the urban economy was in a recovery phase; 'modern rapid urban growth' occurred after the implementation of the opening policy in 1978. This definition provides a morphological perspective on Shanghai's urban evolution and provides the basis of the following analysis.

Table 2. Descriptive statistics of stages in Central Shanghai's urbanisation process

\begin{tabular}{lrrrr}
\hline & $1880 \mathrm{~s}$ & $1940 \mathrm{~s}$ & $1980 \mathrm{~s}$ & $2010 \mathrm{~s}$ \\
\hline Average block size $\left(\mathrm{km}^{2}\right)$ & 0.007 & 0.036 & 0.053 & 0.078 \\
Network density $\left(\mathrm{km} / \mathrm{km}^{2}\right)$ & 17.003 & 8.064 & 8.521 & 6.736 \\
Total street length $(\mathrm{km})$ & 135.208 & 648.515 & $1,713.767$ & $11,591.011$ \\
Total urban area $\left(\mathrm{km}^{2}\right)$ & 7.952 & 80.425 & 201.113 & 1720.759 \\
Population (thousand people) & 302.767 & $6,204.400$ & $9,487.763^{\mp}$ & $21,415.329$ \\
\hline
\end{tabular}

I: The population of the study areas during these periods are estimated according to the reported population densities and the total population. The total population in the $1980 \mathrm{~s}$ and the $2010 \mathrm{~s}$ are $11,859,700$ (1982) and 23,019,148 (2010), respectively. 


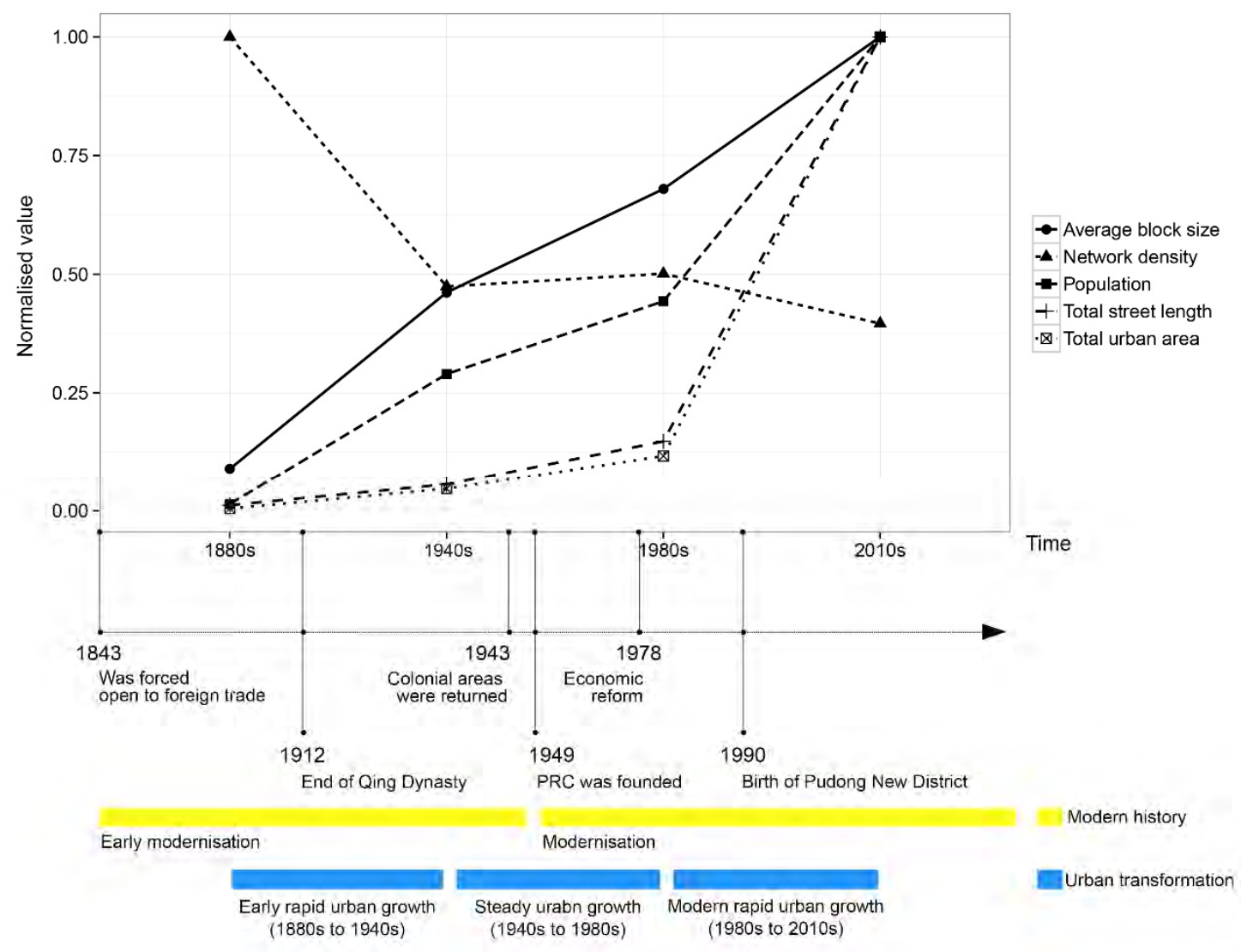

Figure 4. Identification of the critical stages in Shanghai's urbanisation process in terms of the observed spatial and social features (PRC: People Republic of China).

\section{EMPIRICAL RESULTS}

\subsection{Descriptive statistics of the spatio-functional interaction process}

\subsubsection{The overall trend of spatial transformation}

The descriptive statistics of the normalised space syntax centrality measures are shown in Table 3. The method used to generalise these indices is the approach proposed by Hillier and Yang (2012), in which the size effect in the space syntax model is removed at the global scale by taking into account the scaling of the city's size. Average road network connectivity increased over the past 130 years, as did the mean and maximised global integration values, suggesting that the spatial network of Central Shanghai has become more interconnected. In contrast, the changing directions of the average and the maximum choice values are opposite. Along with the increase in the extreme value comes the 
reduction of the average level. This reveals another trend in the urbanisation process: the spatial network turned out to be more hierarchical, and, accordingly, the differentiation among various levels was more significant than before. It is also noteworthy that the growth in average connectivity, the overall integration level and the maximum choice value were interrupted during the period of steady urban growth from the 1940s to the 1980s. The reason for this might be that the self-organisation process dominated temporal urban evolution, which led to the internal growth of the spatial network and a decrease in its hierarchical heterogeneity with the decelerated spatial expansion. The preliminary results indicate that shifts in the spatial centrality structures of cities can provide additional information regarding the stages of urbanisation.

Table 3. Shifting statistical performance of spatial centralities across periods

\begin{tabular}{lcccc}
\hline & $1880 \mathrm{~s}$ & $1940 \mathrm{~s}$ & $1980 \mathrm{~s}$ & $2010 \mathrm{~s}$ \\
\hline Mean connectivity & 4.077 & 4.441 & 3.831 & 4.752 \\
Mean normalised Integration (R10000) & 0.839 & 1.213 & 0.943 & 1.305 \\
Maximum normalised Integration (R10000) & 1.165 & 1.786 & 1.392 & 1.994 \\
Mean normalised Choice (R10000) & 0.965 & 0.994 & 0.892 & 0.501 \\
Maximum normalised Choice (R10000) & 1.505 & 1.555 & 1.497 & 1.635 \\
\hline
\end{tabular}

\subsubsection{The overall functional transforming trend}

During the process of rapid urban growth, the gap between urbanised areas and lessurbanised areas widened; this is supported by evidence of the decline in the mean accessible function density and diversity at all scales, as shown in Table 4. The overall trend of shrinking diversity at the smaller scales (at 1,000 m) was reversed during the process of steady urban growth, when a land-use diversification process occurred. The cognitive effort required by the reachable land-uses at the micro and the mesoscales has become less since the 1940s. This tendency suggests that, since the 1940s, urban function patterns have been more configurationally accessible at scales that are relatively local due to the dominance of the superimposed modern grid-like road system. In contrast, the global mean angular distance to land-use increased. Unlike the interpretation of the spatial centrality process, the performance of land-use systems provides more information regarding the processes of densification, diversification and efficiency optimisation, and 
distinguishes the predefined urbanisation periods in the modernisation of Central Shanghai.

Table 4. Shifting statistical performance of urban function connectivity measures across periods

\begin{tabular}{lrrrr}
\hline & $1880 \mathrm{~s}$ & $1940 \mathrm{~s}$ & $1980 \mathrm{~s}$ & $2010 \mathrm{~s}$ \\
\hline Mean normalised DEN (R1000) & 3.917 & 2.351 & 2.155 & 0.691 \\
Maximum normalised DEN (R1000) & $8.181^{\Uparrow}$ & 7.407 & 8.152 & 9.324 \\
Mean normalised DIV (R1000) & 0.856 & 0.781 & 0.799 & 0.747 \\
Mean DIS (R1000) & 8.209 & 5.651 & 5.739 & 5.985 \\
Max DIS (R1000) & 15.602 & 14.576 & 20.504 & 20.797 \\
& & & & \\
Mean normalised DEN (R5000) & $9.868^{\pi}$ & 7.838 & 5.641 & 2.307 \\
Maximum normalised DEN (R5000) & 9.999 & 10.856 & 10.513 & 10.774 \\
Mean normalised DIV (R5000) & 0.999 & 0.964 & 0.971 & 0.937 \\
Mean DIS (R5000) & 18.289 & 15.814 & 14.895 & 14.471 \\
Max DIS (R5000) & 38.458 & 32.355 & 42.721 & 42.034 \\
& & & & \\
Mean normalised DEN (R10000) & 9.990 & 10.766 & 8.506 & 4.027 \\
Maximum normalised DEN (R10000) & 9.999 & 10.999 & 10.962 & 10.948 \\
Mean normalised DIV (R10000) & 0.999 & 0.997 & 0.995 & 0.976 \\
Mean DIS (R10000) & 18.413 & 18.671 & 23.312 & 21.474 \\
Max DIS (R10000) & 38.724 & 36.311 & 48.694 & 52.615 \\
\hline
\end{tabular}

I: The maximum value, in theory, for urban function connectivity in the $1880 \mathrm{~s}$ is 10 , whereas the limit for other periods is 11 , as a within-group normalised method is applied and the ceiling of the accessible function density is equal to the number of types of active land-uses.

\subsection{Urban centrality maps}

\subsubsection{Space syntax centrality maps}

The space syntax centrality maps present the detailed distributions of street network centralities across scales. The integration distribution at the local scale in the 1880 s shows a separation between the Chinese inner city and the colonial area (Figure 5-a), which reflects the political distance between these two areas. The local centre, which was formed later, combined these two areas and further shaped the current urban centre at the pedestrian level (Figure 5a-d). In regard to the global integration centrality distribution (Figure $5 \mathrm{e}-\mathrm{h}$ ), the streets along the river, which formed the central business area in Shanghai, are highlighted. This area was well known for its national dominance in the import and export trading economy. With the urban spatial expansion, the spatial centre at the global scale shifted to the colonial area, which, until the $1980 \mathrm{~s}$, was further enhanced and expanded towards the west along the main road passing through the east 
and the west. During the 2010s, the global centrality structure indicated by the integration shows growth in the west bank towards the south-west and extends across the river to the newly built developing area on the east bank. The choice maps at the local scales represent, over time, the correlation between integration and choice in general (Figure 5ad), which is due to the significant variation in road network density that is the basic source of urban movements. The choice distributions at larger radii, however, extract the main road system from the less-central context (Figure 6e-h). The highlighted street systems during different periods are highly overlapped, suggesting the dominance of these strongly active routes in directing the urban transformation process. These core streets that are emphasised by the pass-by flows were relatively organically organised before the 1980s but have tended to be more artificial since the modern planned road systems were emphasized. Therefore, the global choice structures in Central Shanghai capture the shifting skeletons of the urbanisation process; while the integration structures illustrate the dynamic potential locations and shapes of the centres along those skeletons, as well as their related scale-dependency.

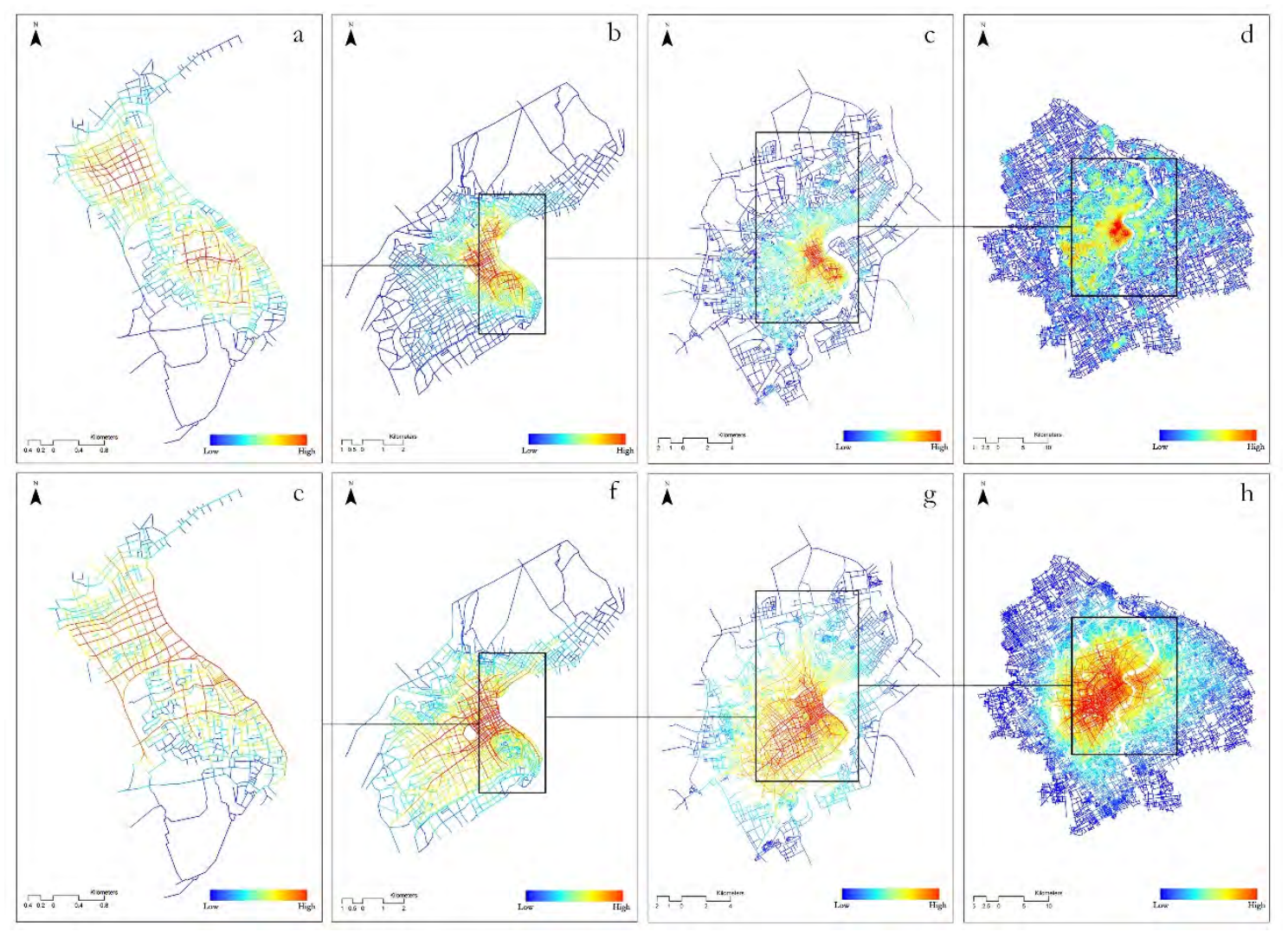

Figure 5. Angular integration maps at 1,000 $\mathrm{m}$ and 10,000 $\mathrm{m}$ across periods ((a, c) $1880 \mathrm{~s}$; (b, f) 1940s; (c, g) 1980s; (d, h) 2010s). 


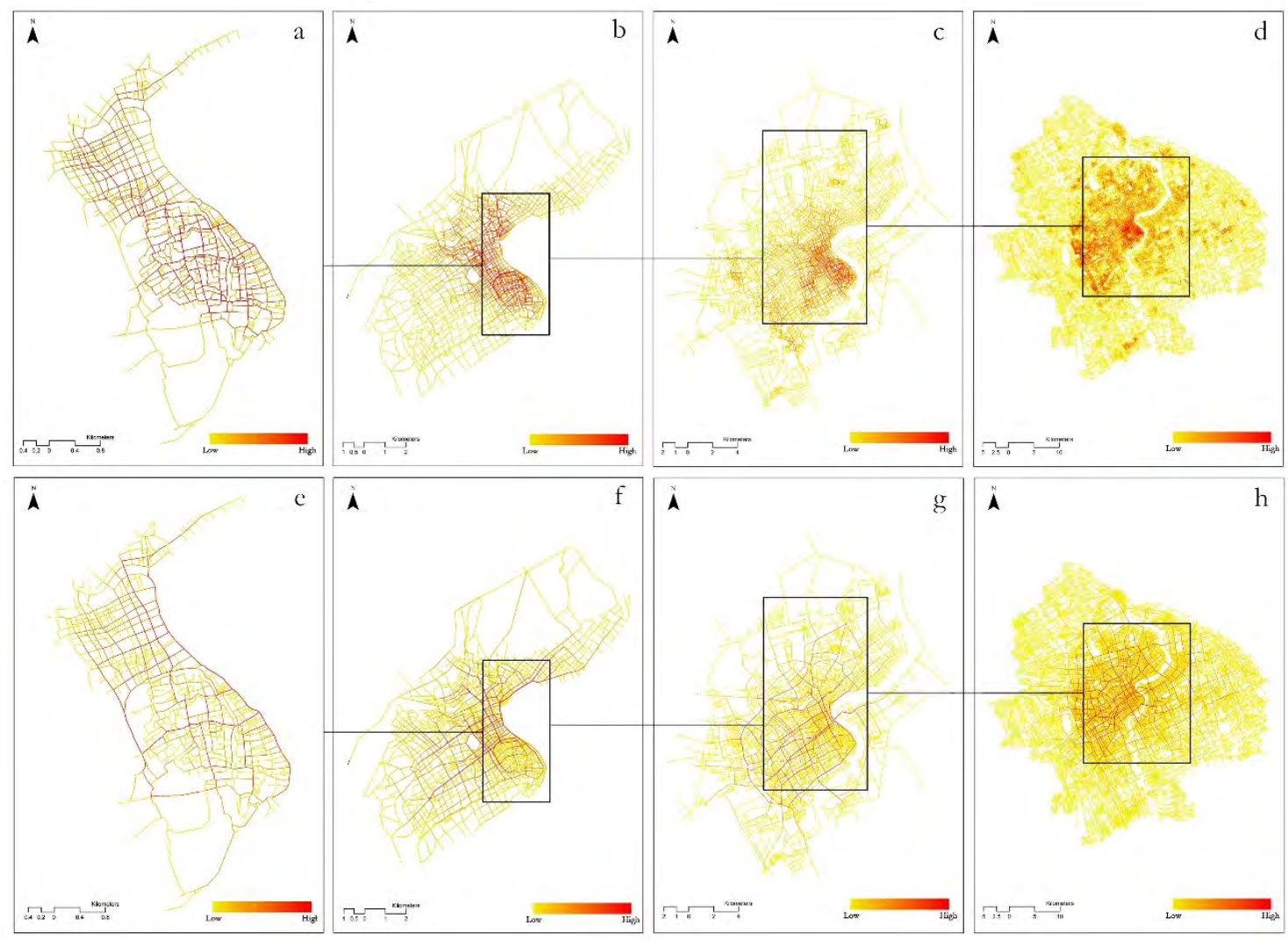

Figure 6. Angular choice maps at 1,000 $\mathrm{m}$ and 10,000 $\mathrm{m}$ across periods ((a, c) 1880s; (b, f) 1940s; (c, g) 1980s; (d, h) 2010s).

\subsubsection{Urban function connectivity and urban function region maps}

Figure 7 maps the integrated urban function connectivity measures at various levels. In the $1880 \mathrm{~s}$, the local function centre structures were relatively isolated from each other along the sides of the river; they were located in the Chinese old town and the colonial area, respectively. This local centre, as indicated by local function connectivity, was moved to the north-west of the inner city in the 1940s, and the newly emergent local functional core has been further consolidated and expanded towards the north and the west since the 1980 s. In the 2010 s, the core of the city's functional centrality structure was anchored in its historical location; simultaneously, the sub-centre structure started to emerge. Some sub-centres have attracted the clustering of various land-uses, thereby forming a polycentric land-use structure at the pedestrian scale. Compared with the spatial centrality structures at the $1,000 \mathrm{~m}$ radius during the same period, the functional centrality structures are visually different in terms of their position, shape, and inherent 
polycentricity. At the global scale, the transformation of the functional structures follows a similar trend that is observed in the shift of spatial centrality through visual judgement. The global functional centre was determined to have been located on the riverside in the $1880 \mathrm{~s}$; it was shifted to the concession areas in the 1940s; it grew towards the west in the 1980s; and it became a convex functional core in the geometric centre of the central urban landscape of today's Shanghai.

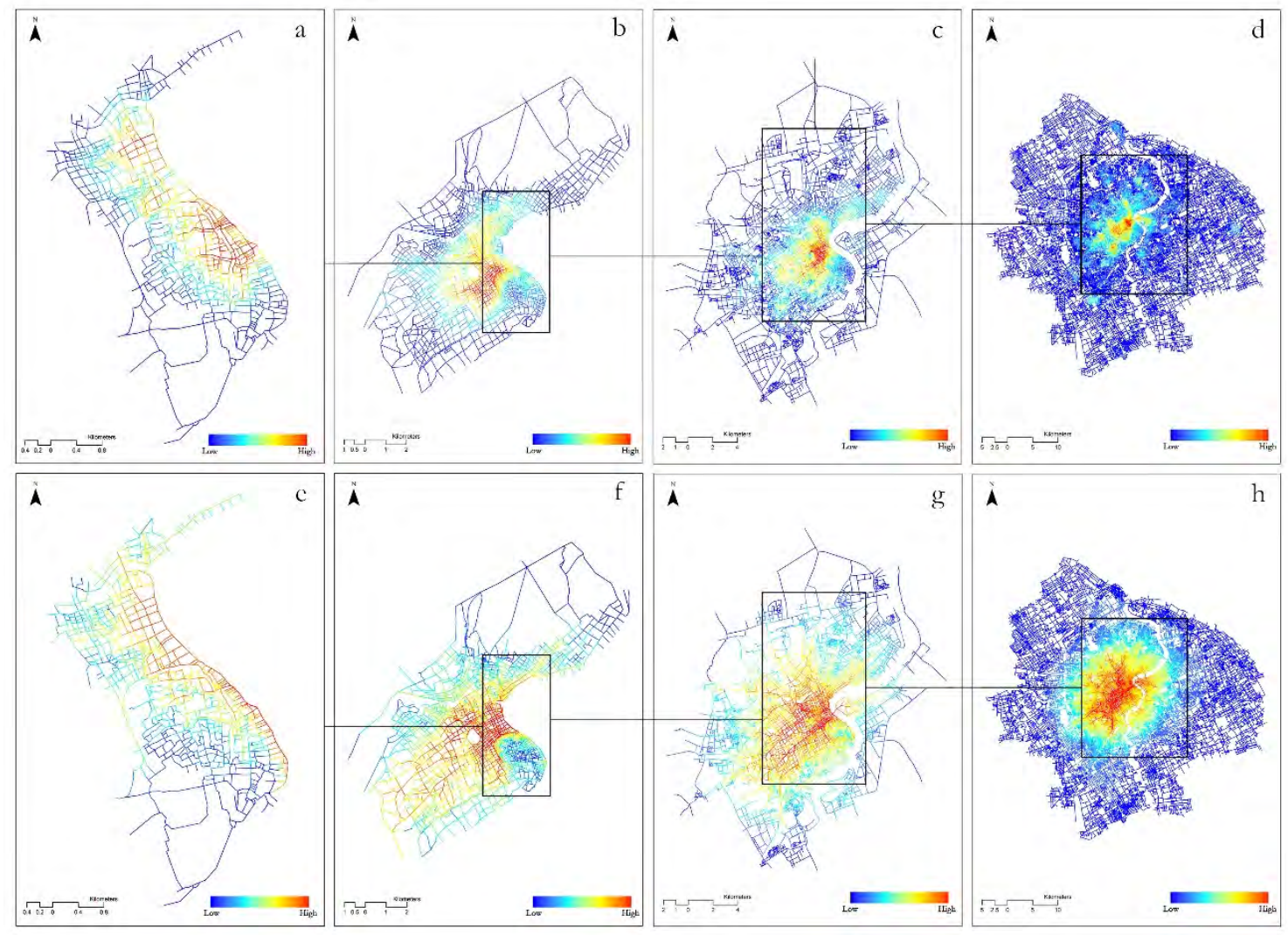

Figure 7. Urban function connectivity maps at 1,000 $\mathrm{m}$ and $10,000 \mathrm{~m}$ across periods ((a, c) $1880 \mathrm{~s} ;(\mathrm{b}, \mathrm{f}) 1940 \mathrm{~s} ;(\mathrm{c}, \mathrm{g}) 1980 \mathrm{~s} ;(\mathrm{d}, \mathrm{h}) 2010 \mathrm{~s})$.

As a useful complement to the urban functional centrality structure, the detected urban function regions - based on the statistical performance of the multidimensional function connectivity vectors for different types of land-uses - are mapped in Figure 8. These emergent land-use communities differ from others according to their composition of access to different land-uses. The increase in the number of function regions from the 1880 s to the 2010s suggests that the land-use structures have become more and more complex as urban development has progressed. This complexity is also reflected by the 
presence of some specific land-use communities that are led by some specific type(s) of land-uses. In the 1880s, the daily active streets (C2), including most of the areas in the Chinese old town and the small area connecting it with the colonial district, were places with better connectivity to cultural amenities, hotels, parks and recreation establishments. This makes sense because during that period the Chinese inner city maintained many temples and destinations for daily entertainment purposes. The colonial area at this time contained the developed business areas (C1), led by modern schools, healthcare and other public services. These two land-use communities were in close proximity to the central business area $(\mathrm{C} 4)$ on the riverside. The differentiation between the colonial area and the Chinese old inner city provides evidence of the political variation between these two places, illustrating that land-use patterns are also products of the political landscape and confirming that the statistical characteristics of the structure of these urban functional regions have spatial meanings. During the 1940 s, the urban functional region structures shifted; two developing areas (C4 and C5) emerged and were led by the newly planned educational facilities but distinguished by being mixed with other land-uses. Urban functional expansion during this period was education-oriented. The central business area (C3) in the 1980s was larger than that in the 1940s because the developed business area of the 1940s was upgraded and merged with it. Other functional regions at this time were distinguished based on the overall degrees of spatial co-presence among the active landuses, with the exception of two types of developing areas (C2 and C4) with lower overall levels of land-use mixtures, as highlighted by the dominance of their connectivity to transport facilities and community parks. Therefore, after the opening policy was implemented, growth in urban functions was led by transport services and parks in residential communities. In regard to the 2010s, there were seven function regions detected in the clustering analysis. Moreover, the discrimination among them was fundamentally influenced by the presence of ensemble connectivity to various land-uses. However, some patches (C6) emerged in a fragmented manner with the dominance of educational services, i.e., the vast campuses of the universities. The location of the central business district (C2) was anchored in the same place as 30 years ago. The less urbanised areas $(\mathrm{C} 1, \mathrm{C} 5$ and $\mathrm{C} 7)$ were highlighted by their connectivity to the public urban facilities, such as transport, hospitals and educational institutions, which implies that essential urban services, as vital public goods, were more important than other urban functions in the developing areas. This suggests that requirements for health care and travel 
convenience have become additional demands of modern society, much like educational functions in the pre-modern society of Central Shanghai. These shifting patterns in the urban function regions create a detailed picture of the dynamic changes in the boundaries and structures of land-use communities; but more importantly, they capture changes in the determination of urban function connectivity.

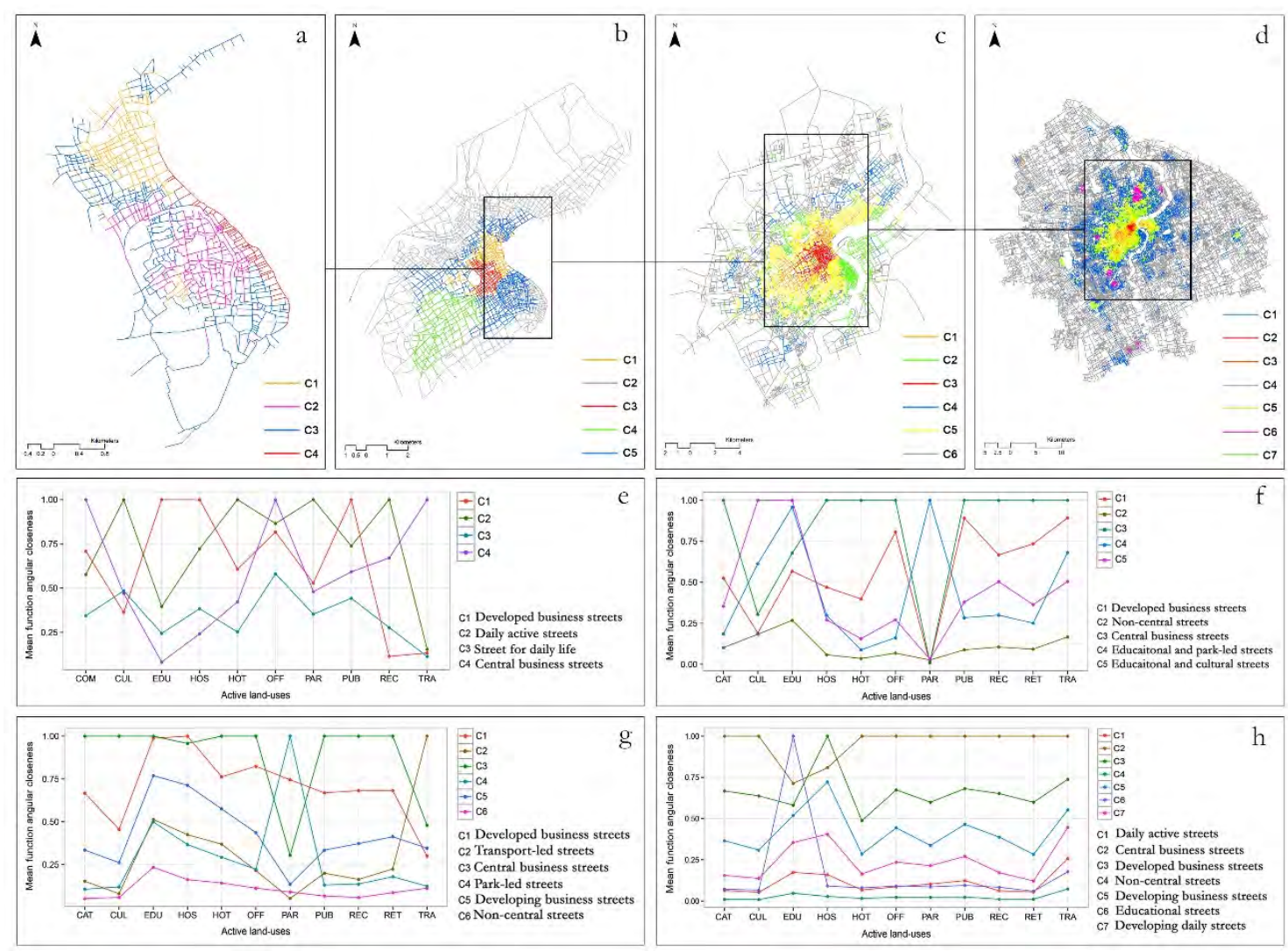

Figure 8. Urban function region maps across periods ((a) 1880s; (b) 1940s; (c) 1980s; (d) 2010s) and the average function angular closeness index for each active land-use in urban function regions across periods ((e) 1880s; (f) 1940s; (g) 1980s; (h) 2010s).

\subsubsection{Centrality change detection}

The urban growth patterns reflected by changes in global integration structures are shown in Figure 9a-c. The street-based results are rasterised because of the existence of the spatial bias within historical street network data. During the early rapid urban growth process, the spatial advantage of the colonial area increased, whereas that of Shanghai's old town decreased. During the steady urban growth stage in the 1940s, global integration centrality moved to the outskirts, where some centres were emerging, including the area 
around the railway station and the Xujiahui business areas. The cost of the spillover of this integration centrality was the decrease of centrality in the colonial area. This observation reveals that the gravity core of the newly emerging city centre moved to the area where the road network densification process occurred. During the modern rapid urban growth process, the city's central area - indicated by higher integration values became stable, with very few changes in global integration centrality. Nevertheless, this urban expansion resulted in the dramatic growth of spatial advantages on the city's periphery in the 1980s. The new Pudong district, for instance, maintained the grouped enhancement of the network shallowness, which suggests that it could easily facilitate further growth. Another two centres that were planned in the 1980s - the Wujiaochang and Tianlin areas - are also captured. In this case, the steady urban growth process contributed to the expansion of the city's central area and the shift of its gravity core and consolidated the city centre, which maintained its centrality during the process of modern rapid urban growth. These results imply that rapid urban growth might not naturally lead to an immediate shift in the centrality structure, but the steady urban growth and spatial densification that follow it will lead to such a shift and thus strengthen the city centre's position in the future.

Normalising global urban function connectivity enables comparisons across cities with different sizes of land-uses to represent the shifting flows of land-use centrality structures (Figure 9d-f). During early rapid urban growth, urban functional centrality moved to the colonial area and the south-west from the Chinese old town. When steady urban growth began, the functional centrality in the north (around the railway station and the Changshu Road) significantly increased, and the areas around Xujiahui were also expanded. Most of the hinterland and the Chinese old town were also functionally strengthened. These findings confirm the occurrence of internal growth during this period, as indicated in the discussion above. After the opening policy was allocated nationally, the Pudong district started to obtain political and planning support, and, therefore, it experienced a significant improvement in its global function connectivity. The Tianlin districts also demonstrated an agglomeration of new land-uses. The earliest colonial area, however, lost its accumulated functional centrality due to the shift of the gravity core. 


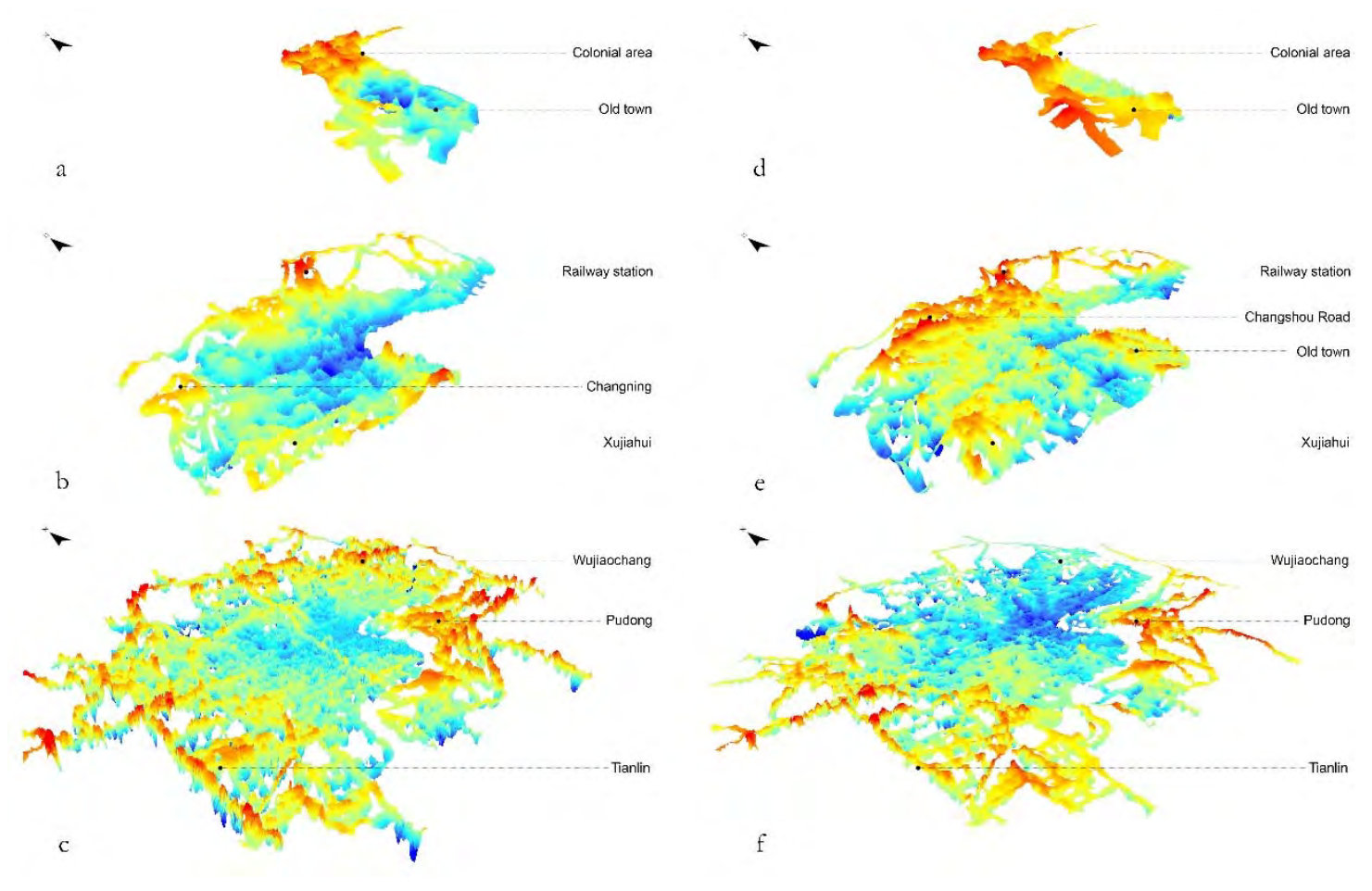

Figure 9. Global centrality change. (Global integration change and global urban function connectivity change during the early rapid urban growth stage between the 1880 s and the 1940s ((a) and (d)), during the steady urban growth stage between the 1940s and the 1980s ((b) and (e)), and during the modern rapid urban growth stage between the 1980s and the 2010s ((c) and (f)). (Red denotes an increase in centrality whereas blue denotes a decrease).

\subsection{Inter-scale and inter-centrality correlation}

The interplay between the integration and the choice centrality measures at various scales across time is further quantitatively scrutinised based on comparing the correlation matrices between them, and the related results are illustrated in Figure 10. The most obvious trend that appears in all the historic snapshots is the inter-scale correlation for a certain type of spatial syntax centrality. The angular integration values at various radii are highly correlated; the choice metrics at different levels are also strongly interrelated, with the exception that the interdependence between local choice and global choice is significantly weaker. The correlation between different centrality measures, however, varies according to the different development stages of Shanghai's urbanisation. The synergy between the spatial centrality indices of the spatial network system, indicated by the inter-centrality correlation, became significantly weaker from the 1980s onward, particularly at the higher spatial levels. This is the consequence of the modern road 
system's invasion of the historical spatial grids, which further intensified the division between the integration and choice structures.
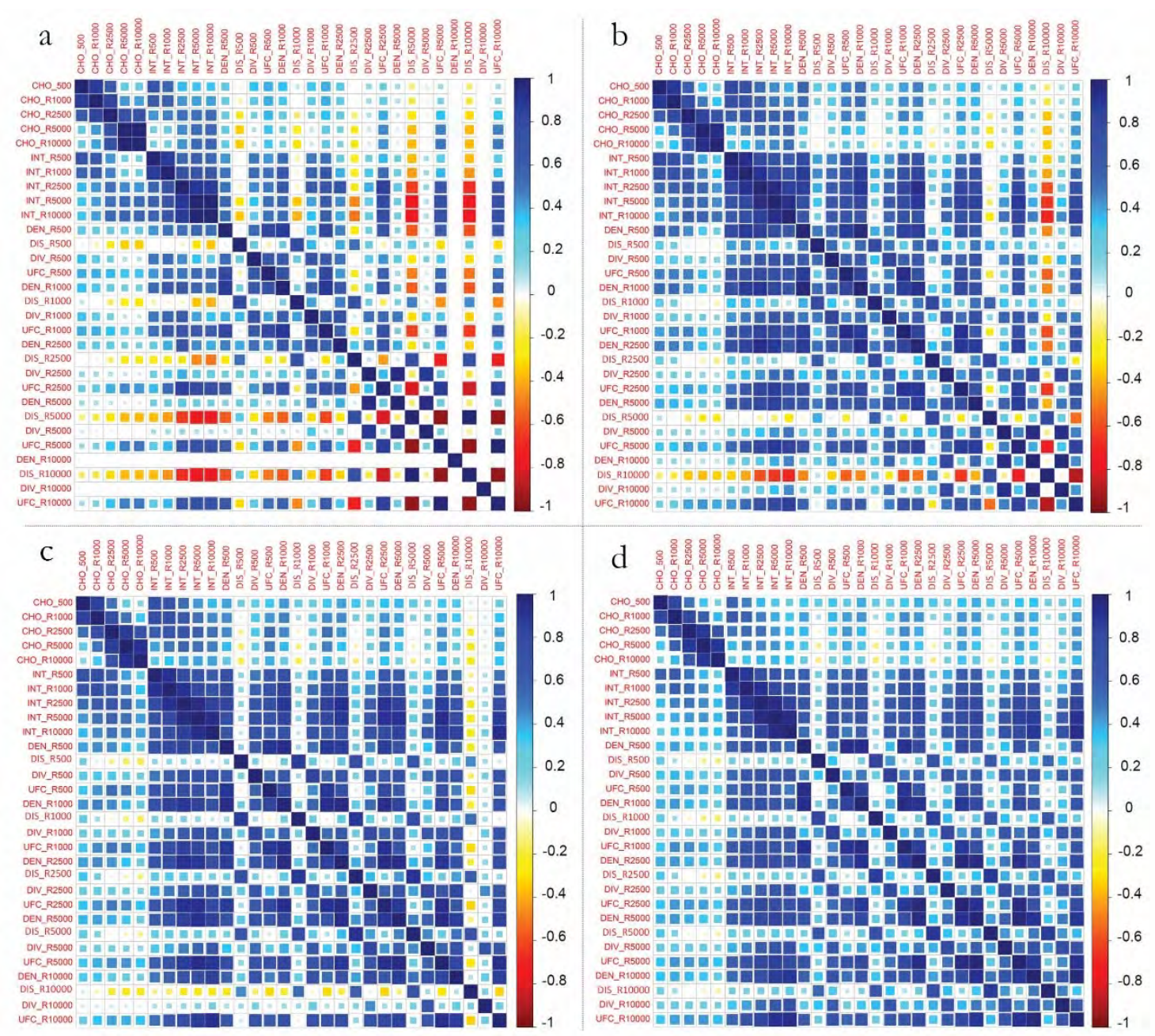

Figure 10. Correlation matrices between any two pairs of urban centralities (integration (INT), choice (CHO), density (DEN), diversity (DIV), cognitive distance (DIS) and the integrated urban function connectivity (UFC)) across various radii during different periods ((a) 1880s; (b) 1940s; (c) 1980s; (d) 2010s). Note: the standard deviation of accessible function density and diversity is 0 for Central Shanghai in the $1880 \mathrm{~s}$.

The results of the correlation matrices among the functional centrality measures for the land-use system are reported. A general trend here is that the accessible function density and the integrated urban function connectivity were strongly correlated with a positive sign at all scales, which illustrates the importance of the basic scale effects in measuring the land-use interaction. Furthermore, the function density and diversity exhibit a positive relationship across scales. Historically, the significance of this density- 
diversity synergy became more and more significant at different scales, and specifically, the local diversity structures have been recognised by the land-use density distributions at the larger scales more successfully since the 1940s (compared to earlier) thereby generating local-global synergy between density and diversity. The mean angular step depth to the nearby functions is positively related to the land-use clustering and mixture degrees at the local levels, but this relationship is reversed when a critical radius is reached. This implies that the urban activities are clustered locally in the urbanised area, requiring more cognitive efforts to interact at the lower scales but less angular step depth to interact at the city-wide scales. The critical scale for the conversion of the relationship between the average angular cost and the reachable land-use density was different across cases during the different historical periods. The critical scale was the radius of $2500 \mathrm{~m}$ in the $1880 \mathrm{~s}, 5,000 \mathrm{~m}$ in the $1940 \mathrm{~s}, 10,000 \mathrm{~m}$ in the $1980 \mathrm{~s}$ and $150,000 \mathrm{~m}$ in the $2010 \mathrm{~s}$ (not shown in the figure), respectively. The increase in the critical radius across time is the consequence of the increasingly rapid urban expansion that has caused Central Shanghai to continuously grow larger and larger.

\subsection{Discriminant analysis: delineating urban function regions}

The first two canonical discriminating functions in all the samples can be classified into two types: the optimum and the sub-optimum spatio-functional synergy, represented by the different degrees of co-presence between spatial and functional centralities across scales. The meanings of each predictor function are explained by recording the significant loadings of the centrality measures (Table 5). Function 1 is the optimum spatio-functional centrality, mostly defined by the most significant co-presence between centralities across scales, which is visually close to the global centrality structure; while function 2 is the sub-optimum spatio-functional interaction with a lower degree of centrality co-presence. Figure 11 plots the detected urban function regions against the two core functions. Notably, the urban regions can be successfully discriminated by the two principal functions. With urban development, the range of the two functions becomes wider, which indicates that the urban centrality structures have been historically polarised. Although the picture clearly shows shifts in the urban function regions' centroids over time, some similarities can be easily identified. The central business streets and the developed business streets score high on optimum spatio-functional centrality but low on suboptimum spatio-functional interaction. The non-central streets, as expected, are scored 
negatively in both cases. The streets dominated by a specific type(s) of land-use(s) are highlighted with positive scores on function 2 but negative or minuscule positive values for function 1 . The developing business streets, or the daily active streets, are placed in the second quadrant, where both functions are scored positively. The consistency of the relative positions of different function regions in the scatter diagrams reveals that the complex spatio-functional interaction is the main factor impacting the streets' typology in terms of the land-use patterns.

Table 5. Structure matrix table in Central Shanghai during various periods ((a) 1880s; (b) 1940s; (c) 1980s; (d) 2010s).

\begin{tabular}{|c|c|c|c|c|c|c|c|c|}
\hline & \multicolumn{2}{|c|}{$1880 \mathrm{~s}$} & \multicolumn{2}{|c|}{ 1940s } & \multicolumn{2}{|c|}{$1980 \mathrm{~s}$} & \multicolumn{2}{|c|}{$2010 \mathrm{~s}$} \\
\hline & $\mathrm{CF} 1$ & $\mathrm{CF} 2$ & $\mathrm{CF} 1$ & $\mathrm{CF} 2$ & $\mathrm{CF} 1$ & $\mathrm{CF} 2$ & $\mathrm{CF} 1$ & $\mathrm{CF} 2$ \\
\hline \multicolumn{9}{|c|}{ Space syntax variables } \\
\hline INT_R500 & & & 0.429 & & 0.418 & 0.232 & 0.352 & \\
\hline INT_R1000 & 0.221 & 0.262 & 0.606 & & 0.576 & 0.291 & 0.483 & 0.116 \\
\hline INT_R2500 & 0.448 & 0.144 & 0.763 & & 0.708 & 0.313 & 0.541 & 0.261 \\
\hline INT_R5000 & 0.466 & & 0.639 & 0.302 & 0.677 & 0.391 & 0.516 & 0.426 \\
\hline INT_R10000 & 0.453 & & 0.511 & 0.390 & & & 0.449 & 0.578 \\
\hline CHO_R500 & & & 0.229 & & 0.173 & 0.220 & 0.143 & \\
\hline CHO_R1000 & 0.184 & 0.357 & 0.241 & & 0.236 & 0.154 & 0.203 & \\
\hline CHO_R2500 & 0.272 & 0.151 & 0.255 & & 0.275 & & 0.209 & \\
\hline CHO_R5000 & 0.412 & -0.177 & 0.203 & & 0.246 & & 0.188 & \\
\hline $\mathrm{CHO} \mathrm{R} 10000$ & 0.417 & -0.199 & & & 0.188 & & 0.150 & 0.115 \\
\hline \multicolumn{9}{|c|}{ Urban function connectivity variables } \\
\hline DEN_R500 & & & 0.708 & & 0.775 & & 0.685 & -0.341 \\
\hline DEN_R1000 & & & 0.788 & & 0.847 & & 0.873 & -0.320 \\
\hline DEN_R2500 & 0.466 & 0.463 & 0.703 & 0.106 & 0.757 & 0.230 & 0.771 & \\
\hline DEN_R5000 & & & 0.377 & 0.270 & 0.611 & 0.517 & 0.677 & 0.360 \\
\hline DEN_R10000 & & & 0.087 & 0.152 & 0.359 & 0.526 & 0.494 & 0.563 \\
\hline DIV_R500 & 0.424 & 0.265 & 0.273 & 0.445 & 0.377 & 0.525 & 0.323 & 0.560 \\
\hline DIV_R1000 & 0.324 & 0.343 & 0.200 & 0.298 & 0.305 & 0.395 & 0.240 & 0.443 \\
\hline DIV_R2500 & 0.110 & & 0.151 & 0.233 & 0.268 & 0.364 & 0.254 & 0.407 \\
\hline DIV_R5000 & 0.034 & & 0.118 & 0.213 & 0.186 & 0.292 & 0.280 & 0.432 \\
\hline DIV_R10000 & & & & 0.145 & & & 0.184 & 0.375 \\
\hline \multicolumn{9}{|l|}{ DIS_R500 } \\
\hline DIS_R1000 & & 0.484 & & -0.193 & & & & \\
\hline DIS_R2500 & -0.107 & 0.591 & & -0.287 & & 0.133 & & \\
\hline DIS_R5000 & -0.609 & 0.387 & & -0.208 & & 0.214 & & \\
\hline DIS_R10000 & -0.634 & 0.365 & -0.442 & -0.320 & -0.104 & 0.134 & & 0.067 \\
\hline
\end{tabular}

Note: INT - Integration; CHO - Choice; DEN: - Accessible function density; DIV: Accessible function diversity; DIS: Mean angular distance to functions; CF1: The optimum spatio-functional centrality; CF2: The sub-optimum spatio-functional centrality. 

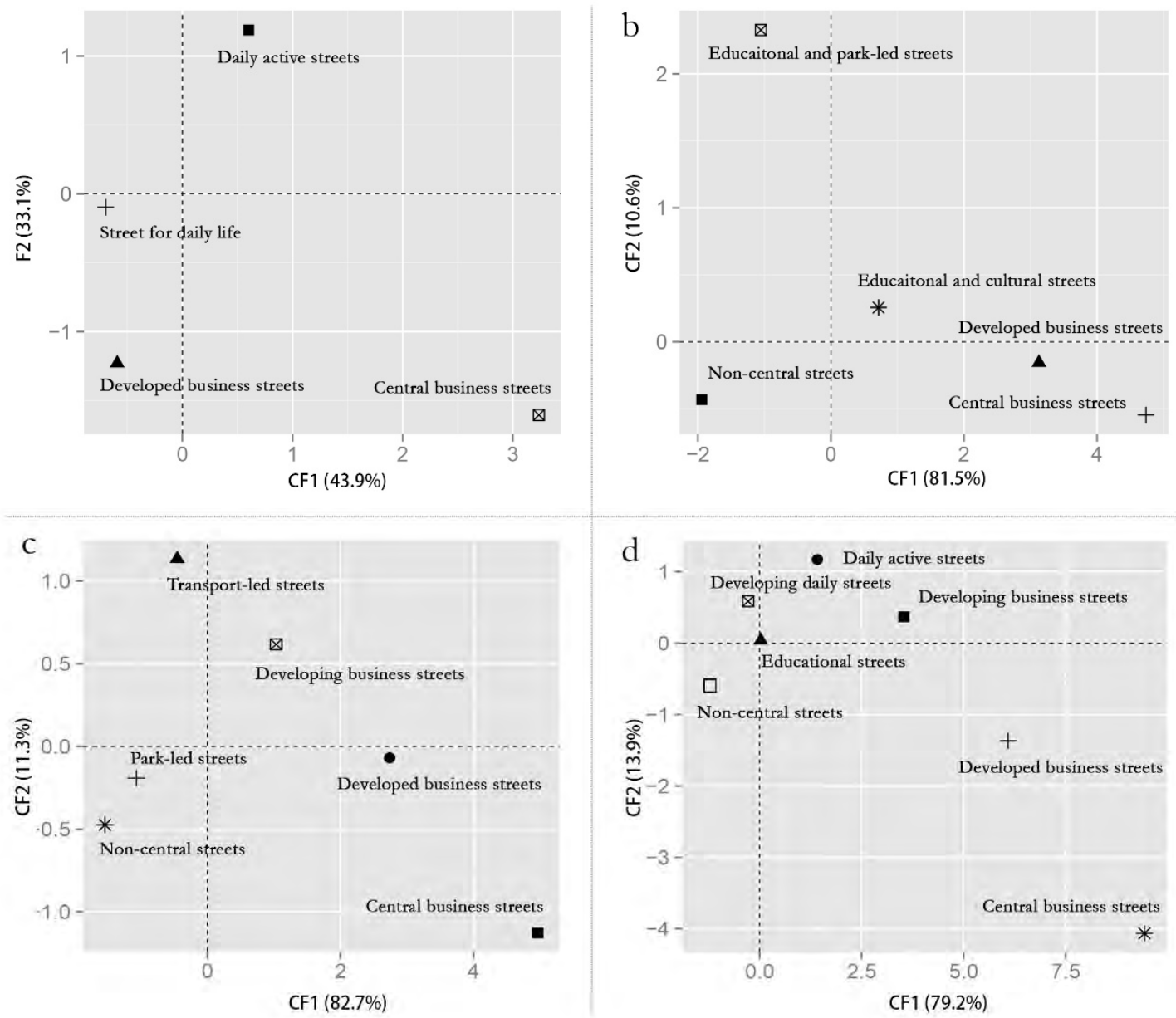

Figure 11. Canonical discriminant functions at urban function region centroids ((a) 1880s; (b) 1940s; (c) 1980s; (d) 2010s).

To further evaluate the performance of these two detected functions in predicting the memberships of the urban function regions, the second step of the discriminant analysis is to assess the predictability of the projected variables in terms of segmenting the known region structures. The relevant results are reported in Table 6. There are three specifications of models utilised for comparison: the first model is organised with only the spatial centrality variables, the second one only contains the functional centrality factors, and the last one is a mixed model with both families of the structural centralities proposed in this article. The accuracy of the models with only the space syntax variables during different periods is more than half (except for the model of the 1980s) with an overall accuracy of $47.1 \%$. The highest predictability of the spatial centrality variables appeared in the 1940s, when early rapid urban growth occurred. Compared with the performance of the spatial centrality variables, the urban function connectivity variables maintain higher accuracy in all samples. This is consistent with our expectations, as the 
land-use regions are inevitably impacted by the land-use system in some sense. The detected urban function regions here, however, are treated as the independent classification of the streets because they are defined based on the combined statistical significance of the connectivity for diverse land-uses rather than the urban function connectivity indices for all the land-uses that are used to predict the affiliation of function regions. More importantly, the extent to which the spatial centrality measure will improve the predictive model with only the functional centrality measures is the primary concern of the designed discriminant analysis. The irreplaceable role that urban form plays in discriminating among urban function regions is verified by the observation of the improved model's predictive accuracy when the spatial centrality measures are taken into account in the benchmark models. This also provides evidence that the spatial and functional centrality structures not only preserve their similarities but also sustain a complementary interrelationship, delineating the urban function communities within the urban context. Moreover, it is arguable that other factors might also be strongly influential, particularly during the periods after the implementation of the open policy when the predictability of the spatio-functional interaction is smaller than that during the previous period.

Table 6. Prediction accuracy of urban function regions using space syntax centrality and urban function connectivity measures across scales

\begin{tabular}{|c|c|c|c|c|c|c|c|c|}
\hline & \multicolumn{7}{|c|}{ Prediction accuracy for regions } & $\begin{array}{l}\text { Overall } \\
\text { accuracy }\end{array}$ \\
\hline Membership & 1 & 2 & 3 & 4 & & & & \\
\hline SSX variables & $57.6 \%$ & $63.1 \%$ & $56.3 \%$ & $39.9 \%$ & & & & $57.4 \%$ \\
\hline UFC variables & $71.8 \%$ & $86.4 \%$ & $76.1 \%$ & $84.5 \%$ & & & & $79.4 \%$ \\
\hline SSX and UFC variables & $78.2 \%$ & $90.6 \%$ & $81.9 \%$ & $89.1 \%$ & & & & $86.7 \%$ \\
\hline \multicolumn{9}{|l|}{$1940 \mathrm{~s}$} \\
\hline Membership & 1 & 2 & 3 & 4 & 5 & & & \\
\hline SSX variables & $50.6 \%$ & $75.0 \%$ & $64.4 \%$ & $76.2 \%$ & $67.6 \%$ & & & $69.3 \%$ \\
\hline UFC variables & $68.3 \%$ & $83.2 \%$ & $84.4 \%$ & $83.9 \%$ & $63.6 \%$ & & & $75.7 \%$ \\
\hline SSX and UFC variables & $74.4 \%$ & $85.2 \%$ & $86.1 \%$ & $80.7 \%$ & $71.7 \%$ & & & $80.6 \%$ \\
\hline \multicolumn{9}{|l|}{$1980 \mathrm{~s}$} \\
\hline Membership & 1 & 2 & 3 & 4 & 5 & 6 & & \\
\hline SSX variables & $54.3 \%$ & $33.0 \%$ & $76.9 \%$ & $38.3 \%$ & $27.1 \%$ & $56.0 \%$ & & $47.1 \%$ \\
\hline UFC variables & $62.6 \%$ & $61.3 \%$ & $74.6 \%$ & $61.1 \%$ & $53.1 \%$ & $51.5 \%$ & & $55.9 \%$ \\
\hline SSX and UFC variables & $70.0 \%$ & $58.5 \%$ & $74.7 \%$ & $65.7 \%$ & $55.5 \%$ & $55.9 \%$ & & $61.1 \%$ \\
\hline \multicolumn{9}{|l|}{$2010 \mathrm{~s}$} \\
\hline Membership & 1 & 2 & 3 & 4 & 5 & 6 & 7 & \\
\hline SSX variables & $40.9 \%$ & $80.1 \%$ & $38.3 \%$ & $75.4 \%$ & $28.2 \%$ & $80.1 \%$ & $37.6 \%$ & $59.6 \%$ \\
\hline UFC variables & $50.1 \%$ & $74.3 \%$ & $62.4 \%$ & $78.6 \%$ & $55.3 \%$ & $74.3 \%$ & $59.2 \%$ & $67.5 \%$ \\
\hline SSX and UFC variables & $51.6 \%$ & $74.3 \%$ & $63.9 \%$ & $79.5 \%$ & $56.5 \%$ & $74.3 \%$ & $58.2 \%$ & $72.9 \%$ \\
\hline
\end{tabular}




\section{SUMMARY AND DISCUSSION}

This study sheds light on the evolutionary nature of the interaction between urban forms and functions from a syntactic perspective using a series of quantitative measurements of urban centrality structures with historical street network and POIs datasets. By introducing centrality indices for spatial and functional morphologies, namely the space syntax centrality indices and the urban function connectivity metrics, generated by the spatial network and land-use patterns in tandem, this article detects and compares the spatial and functional centrality processes and their interactions at different stages of the urbanisation process. This is one approach to integrating historical spatio-functional information and extracting comprehensive knowledge regarding the urban evolution process. Based on a case study of Central Shanghai, this article summarises a general picture of spatio-functional interaction in the urbanisation process, confirms the novelty of taking into account the function centrality structures in configurational studies and verifies the effectiveness of the detailed measurements. The quantitative framework and associated findings presented here can be used as references to more comprehensively and explicitly describe the dynamics of changing urban centre systems, thus enriching our understanding of urban morphological evolution and supporting appropriate planning and design applications. Supported by the centrality structures produced by the methods introduced, urban designers and planners can access the cooperation between the proposed spatial layouts and the land-use allocation within the spatial and functional contexts. Moreover, unfolding the effects of the land-use change on the shift of configurational centrality structure without the change of built form may be particularly helpful for the preservation of the historic districts where the built environment is strictly managed. It yields that this 'soft' intervention can be adopted to sustain the functionality of historic areas through empowering the connectivity of local land-use patterns with sufficient respect to the historic built forms.

This empirical study of Central Shanghai's urbanisation history implies that the urban evolution process can be jointly characterised by the spatial and functional centralities of the urban context. There are several aspects of its potential contribution. First, it suggests that urban form interacts with urban function by shaping the interrelated spatial and function centralities. They are not static but rather dynamic, as parts of a complex process. The defined urbanised stages in history can be successfully characterised by the ways in which the spatial and function elements are interrelated. 
Furthermore, the proposed functional centrality structures capture various principal aspects of the configurational agglomeration of active land-uses through the street network, such as the densification, diversification and distanciation processes, which are different from those informed by the spatial centre's patterns captured by the space syntax centrality, particularly at the local scales. The current study provides theoretical propositions and methods for modelling the syntactic properties of a land-use system and spatial network in a morphological analysis, and in space syntax studies in particular. Moreover, this research demonstrates that urban spatial centralities influence functional centralities in a complex and comprehensive fashion, which has been captured by the integrated urban function connectivity measure that summarises the interplay among individual functional centrality measures. Additionally, it reveals that rapid spatial expansion does not naturally lead to a change in the structure of urban centres, but functional expansions with spatial consolidation result in the reorganisation of the hierarchy of centres. The results of the discriminant analysis (of predicting the membership of the detected urban function regions) quantitatively verified the complementarity between urban form and functions.

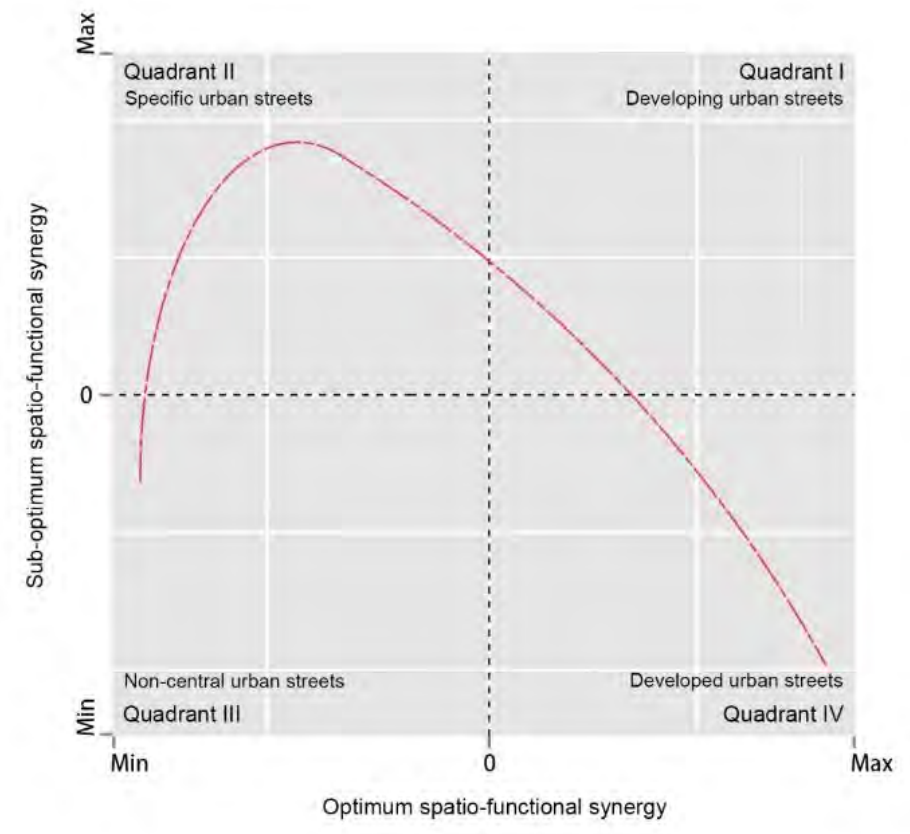

Figure 12. A generalised model for the distinction of the street-based land-use community structures based on the integration between the optimum and the suboptimum spatio-functional synergy functions. 
This research also illustrates a general rule that governs how urban streets can be classified by the synergy effects simultaneously exerted by the spatial network and landuse patterns (Figure 12). In such a model, the co-presence of spatial and functional centralities across scales is first packaged as two types of spatio-functional synergies: the optimum and the sub-optimum spatio-functional synergies. The four quadrants in the rectangular coordinate plane defined by these two categories of spatio-functional synergy indicate four classes of urban function regions in streets: the non-central urban streets are located in the third quadrant; the specific urban streets, highlighted by the dominance of connectivity to certain type(s) of land-use(s), are defined in the second quadrant; the developing urban streets, including the daily active streets and the developing business streets, are captured in quadrant I; and the developed urban centres are in quadrant IV. Notably, the locations of function regions in this plane are not only divided by the quadrants in general but also controlled by a curve from quadrant III to quadrant IV, crossing quadrants II and I in a sequence. This curve also represents a process by which urban function regions transform from one type into another due to shifts in the performance of the spatio-functional synergy functions. At different urbanisation stages, the subdivision of these quadrants can vary. During the steady urban growth process, the four quadrants of the plane are relatively balanced, as the deviations arising in both are relatively small. During the rapid urban growth process, however, the polarisation of the spatio-functional synergies will be intensified, which will lead to the compression of the quadrants - an indicator of the gap between the urbanised and non-urbanised areas. The robustness of the emergence of relevant phenomena in the empirical study of Central Shanghai is evidence of the representativeness of the proposed ideal model for segmenting streets based on the complex spatio-functional interaction.

This study provides important insights into the syntactic analysis of the dynamic interaction between urban form and land-use patterns using the historical street network and POIs. The proposed approaches and their results provide references for future studies analysing the urban evolution process. Several areas of this research could be further developed in subsequent efforts. First, the proper weights for the historical POIs could be added into a current framework to infer the popularity of individual function locations for function connectivity computation. In this study, only the POIs in the 2010s are weighted by the assigned social media check-in data; other historical POIs are weighted equally due to the absence of data on their significance. Backed by multiple data sources, the 
explicitness of the delivered functional centrality measures could be enhanced. Second, more empirical experiments could be conducted in the future to validate the generality of our findings and the applicability of our methods across cases. Third, the analysis of the urban evolution process in this work is spatiotemporal with a long time interval. Identifying the evolution of urban functional centrality structures along a fine-grained temporal dimension with emerging geographical location information will be valuable in advancing current knowledge of urban spatial and functional evolution.

\section{REFERENCE}

Agryzkov, T., Martí, P., Nolasco-Cirugeda, A., Serrano-Estrada, L., Tortosa, L. and Vicent, J.F., (2016). Analysing successful public spaces in an urban street network using data from the social networks Foursquare and Twitter. Applied Network Science, 1(1), p.12.

Alonso, W. (1960). A theory of the urban land market. Papers in Regional Science, 6(1), 149-157.

Anas, A., Arnott, R., \& Small, K. A. (1998). Urban spatial structure. Journal of economic literature, 36(3), 1426-1464.

Batty, M. (2009). Accessibility: in search of a unified theory. Environment and Planning B: Planning and Design, 36(2), 191-194.

Batty, M., Couclelis, H., \& Eichen, M. (1997). Urban systems as cellular automata. Environment and Planning B: Planning and Design, 24(2), 159-164.

Bishop, C. M. (2006). Pattern recognition. Machine Learning, 128.

Burger, M., \& Meijers, E. (2012). Form follows function? Linking morphological and functional polycentricity. Urban Studies, 49(5), 1127-1149.

Cataldi, G., Maffei, G. L., \& Vaccaro, P. (2002). Saverio Muratori and the Italian school of planning typology. Urban Morphology, 6(1), 3-14.

Chen, Z., \& Wu, J. (2004). The business atlas of Old Shanghai. Press of Shanghai Academy of Social Sciences.

Christaller, W. (1966). Central places in southern Germany. Prentice-Hall.

Darin, M. (1998). The study of urban form in France. Urban Morphology, 2(2), 63-76.

Eppli, M. J., \& Shilling, J. D. (1996). How critical is a good location to a regional shopping center?. The Journal of real estate research, 12(3), 459.

Geurs, K. T., \& Van Wee, B. (2004). Accessibility evaluation of land-use and transport strategies: review and research directions. Journal of Transport geography, 12(2), 127-140.

Hess, P. M., Moudon, A. V., \& Logsdon, M. G. (2001). Measuring land use patterns for transportation research. Transportation research record, (1780), 17-24. 
Hillier, B. (1996). Cities as movement economies. Urban Design International, 1(1), 4160.

Hillier, B. (1999). Centrality as a process: accounting for attraction inequalities in deformed grids. Urban Design International, 4(3-4), 107-127.

Hillier, B., \& Iida, S. (2005). Network and psychological effects in urban movement. In Spatial information theory (pp. 475-490). Springer Berlin Heidelberg.

Hillier, W. R. G., Yang, T., \& Turner, A. (2012). Normalising least angle choice in Depthmap-and how it opens up new perspectives on the global and local analysis of city space. Journal of Space syntax, 3(2), 155-193.

Horton, F. E., \& Reynolds, D. R. (1971). Effects of urban spatial structure on individual behavior. Economic Geography, 47(1), 36-48.

Hotelling, H. (1990). Stability in competition. In The Collected Economics Articles of Harold Hotelling (pp. 50-63). Springer New York.

Moudon, A. V. (1997). Urban morphology as an emerging interdisciplinary field. Urban morphology, 1(1), 3-10.

Pinho, P., \& Oliveira, V. (2009). Cartographic analysis in urban morphology. Environment and Planning B: Planning and Design, 36(1), 107127.

Pinchemel, P. (1983). Geographers and the city: a contribution to the history of urban geography in France. Academic Press.

Porta, S., Strano, E., Iacoviello, V., Messora, R., Latora, V., Cardillo, A., \& Scellato, S. (2009). Street centrality and densities of retail and services in Bologna, Italy. Environment and Planning B: Planning and design, 36(3), 450-465.

Porta, S., Latora, V., Wang, F., Rueda, S., Strano, E., Scellato, S., \& Latora, L. (2012). Street centrality and the location of economic activities in Barcelona. Urban Studies, 49(7), 1471-1488.

Scoppa, M. D., \& Peponis, J. (2015). Distributed attraction: the effects of street network connectivity upon the distribution of retail frontage in the City of Buenos Aires. Environment and Planning B: Planning and Design, 42(2), 354-378.

Sevtsuk, A. (2010). Path and place: a study of urban geometry and retail activity in Cambridge and Somerville, MA (Doctoral dissertation, Massachusetts Institute of Technology).

Sevtsuk, A. (2014). Location and Agglomeration The Distribution of Retail and Food Businesses in Dense Urban Environments. Journal of Planning Education and Research, 34(4), 374-393.

Shanghai Institute of Surveying and Mapping. (1989). Shanghai business atlas. Shanghai translation press.

Ståhle, A., Marcus, L. \& Karlström, A., (2005). Place Syntax: Geographic accessibility with axial lines in GIS. In Fifth international space syntax symposium (pp. 131144). Techne Press.

Sun, P., Lu, Y., Fu, B., Yang, M., Zhou, J., \& Chai, X. (1999). Shanghai’s planning chronicle. Press of Shanghai Academy of Social Sciences.

Tallon, A. (2013). Urban Regeneration in the UK. Routledge. 
Turner, A. (2001a). Angular analysis. In Proceedings of the 3rd Space Syntax Symposium, Atlanta, University of Michigan.

Waddell, P. (2002). UrbanSim: Modeling urban development for land use, transportation, and environmental planning. Journal of the American Planning Association, 68(3), 297-314.

Wegener, M. (2004). Overview of land-use transport models. Handbook of transport geography and spatial systems, 5, 127-146.

Wheaton, W. C. (1974). A comparative static analysis of urban spatial structure. Journal of Economic Theory, 9(2), 223-237.

Wilson, A. G. (1998). Land-use/transport interaction models: Past and future. Journal of transport economics and policy, 3-26. 